WE-Heraeus Summer School on Nuclear Astrophysics in the Cosmos

\title{
Exotic nuclei
}

\section{Christoph Scheidenberger GSI Darmstadt}




\section{Part 1:}

What are exotic nuclei? Why study? Key questions

\section{Part 2:}

Production and separation of exotic nuclei in the laboratory

\section{Part 3:}

Examples: halo nuclei, 2-proton radioactivity, superheavy elements

Part 4:

Exotic nuclei in nuclear astrophysics

Part 5:

Future opportunities at FAIR 


\section{Introduction}




\section{Pioneering work using mass spectrometry}

Discovery of isotopes



J. J. Thomson

(1913)
High-resolution mass-spectrographs



F. W. Aston ( 1915...1925)

* identification of 212 isotopes

* systematics:

$\rightarrow$ "packing fraction" 


\section{Development of nuclear models}

\section{Discovery of mass excess:}

Masses deviate from whole numbers



\section{First (collective) model:}

Liquid-drop model by C.F.v.Weizsäcker, H. A. Bethe (1935/36)

C. F. v. Weizsäcker

Z. Phys. 96, 431 (1935)

H. A. Bethe

Rev. Mod. Phys. 8, 81 (1936)

$$
\begin{array}{ll}
\text { Volume energy } & \sim A^{(*)} \\
\text { Surface " } & \sim A^{2 / 3} \\
\text { Coulomb " } & \sim-Z^{2} / A^{1 / 3} \\
\text { Asymetry " } & \sim-(Z-A / 2)^{2} / A
\end{array}
$$

(*) $R \sim A^{1 / 3}$

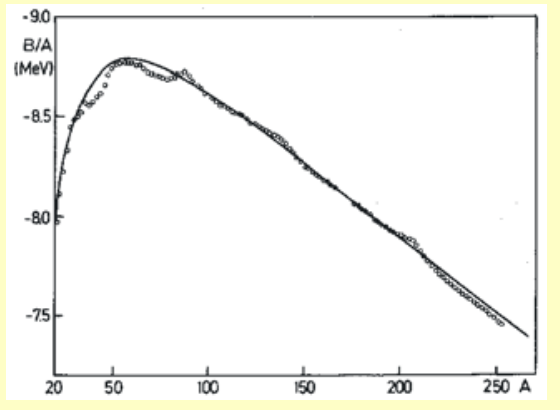




\section{Chart of (stable) nuclei}

-






\section{Shell effect in stable nuclei}



number of stable isotopes



Number of stable isotopes/isotones

---> magic numbers 


\section{Development of nuclear models (II)}

\section{Single-particle shell model (1949):}

Individual properties:

e.g.: excitation energies, magnetic moments

based on Schrödinger equation:

$$
H=\sum_{i}\left[-\left(\frac{\hbar}{2 m}\right) \cdot \Delta_{i i}\right]+\sum_{i<j} V_{i j}
$$

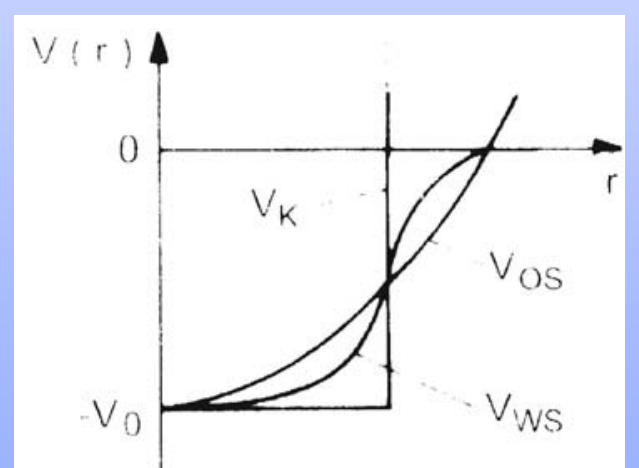






\section{Chart of (known) nuclei}






\section{Nuclear radii do not increase as $A^{1 / 3}$}

1) Nuclear Radius:

Textbooks say: $R \approx 1,3 \mathrm{fm}{ }^{*} A_{0}{ }^{1 / 3}$



.....valid only for nuclei near stability 


\section{Magic numbers depend on $\mathrm{N}$ and $\mathrm{Z}$}

2) Magic numbers:

According to standard textbooks:

$$
2,8,20,28,50,82,126
$$



A. Ozawa et al., PRL 84, 5493 (2000)

New "halo-driven" magic numbers 


\section{Many more bound nuclei exist than anticipated}











\section{Exotic places where they are produced}

Nov. 1986

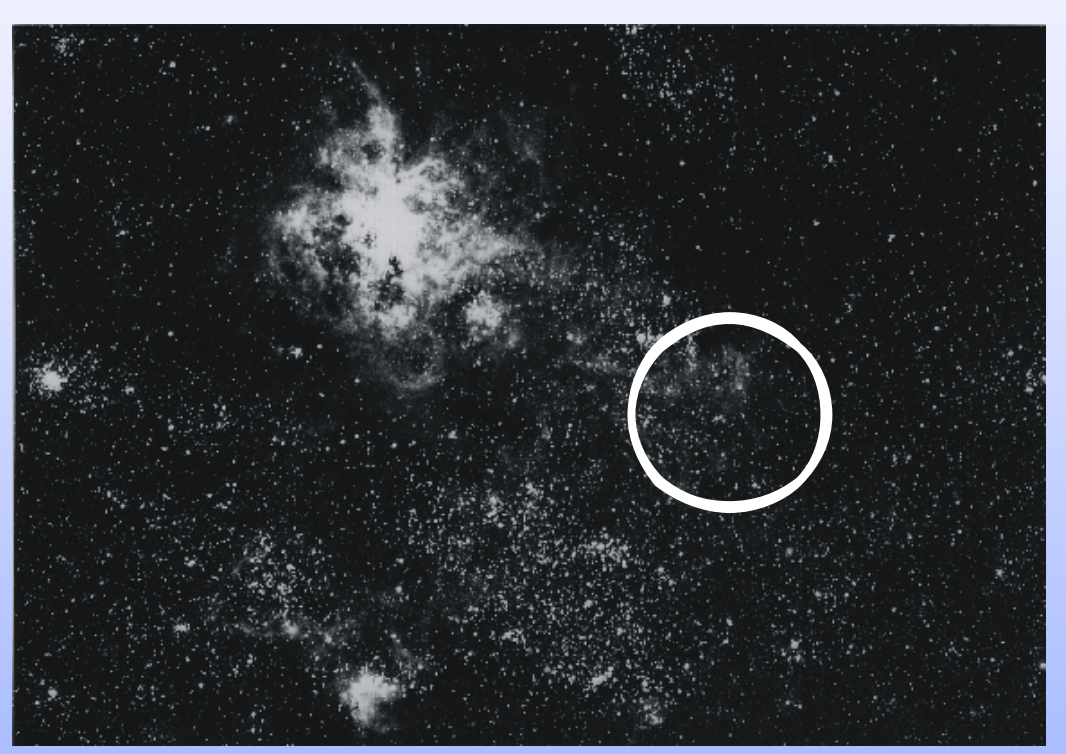

\section{Feb. 1987}



SN1 987A 


\section{What is the meaning of "exotic“}

exotic places

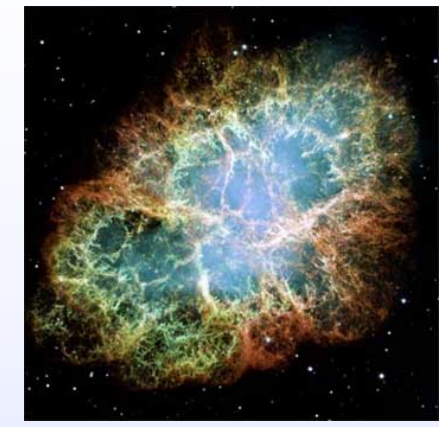

\section{exotic composition}

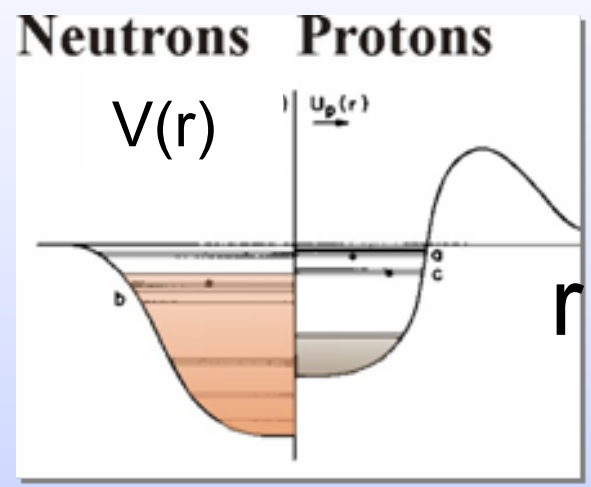

exotic properties

\section{$\rightarrow$ sufficiently many reasons to study exotic nuclei!}




\section{Key questions}

\section{General questions:}

Limits of stability, heaviest elements

Understanding of nuclear forces, isospin dependence

Magicity and shells far-off stability

New phenomena and new decay modes

Nucleosynthesis and elemental abundances

\section{Properties of nuclei:}

"Weight" (mass excess)

"Size" (matter and charge radii)

"Shape" (deformation)

Half-life, decay modes

Electrical and magnetic moments, spins

Single-particle structure

Collective phenomena (giant dipole resonance) 


\section{Challenge: understand solar system element and mass abundances}



Where, when and how are the elements produced?

Understand the observed distribution, qualitatively and quantitatively!

Why no elements $Z>92$, why no masses $A>240$ ? 




Hubble Space Telescope



Apache Point
E.g., discover and understand the formation of the first stars and galaxies, chemical evolution of galaxies, measure the geometry of the Universe and the distribution of (dark) matter, investigate the evolution of galaxies and the production of elements by stars, and the process of star and planet formation



Cowan et al., NIC-9 proceedings 


\section{Radioactive nuclei tell us: elements are synthesized in stars}

${ }^{26} \mathrm{Al}$ half-life $7.8 \times 10^{5} \mathrm{y}$ Stars are still making atoms A'\&A 298 (June, 1995) 445 


\section{Element synthesis processes}

- Big Bang Nucleosynthesis

- pp-chain

- CNO cycle

- Helium, C, O, Ne, Si burning

- s-process

- $r$-process

- rp-process

- vp - process

\section{Radioactive ("exotic") nuclei}

- $p$ - process

- $\alpha$ - process

- fission recycling

- Cosmic ray spallation

- pyconuclear fusion

$\bullet+$ others 



\section{We can look into the interior of stars!}




\section{Production}


Fragmentation, spallation



Coulomb dissociation, fission



Compound nuclei, fusion






\section{Production reactions}






\section{Technical concepts to produce exotic nuclei}



$\begin{array}{ll}\text { Elements } & \text { Universal } \\ \text { Separation time } & <1 \mathrm{~ms} \\ \text { Selectivity } & \text { pure beams } \\ \text { Intensity } & \text { moderate } \\ \text { Energy of } & 50 . . .1500 \mathrm{~A} \mathrm{MeV} \\ \text { secondary beam }\end{array}$

Options



Chemically difficult

$0.1 s \sim$

contaminants possible

high

\section{Hybrid (in-flight + ISOL)}



Universal (?)

$\sim \mathrm{ms}$

pure beams (?)

moderate

$$
10-100 \mathrm{keV}
$$

Post-Acceleration (1....10 A MeV) 


\section{World view of radioactive-beam facilities}






\section{The exotic beam facilities at GSI}

Laboratory tour at GSI: Thursday afternoon 


\section{Production of exotic nuclei by projectile fragmentation}



fragmentation, invented at LBNL in the 1980's



$$
\begin{array}{rr}
\text { Produktionsraten: } 10^{5} / \text { sek. } \ldots . & 10^{-5} / \text { sek. } \\
& (\approx 1 / \text { Tag })
\end{array}
$$




\section{Spallation and fission of uranium}











\section{Nucleo"synthesis" by spallation of cosmic rays}
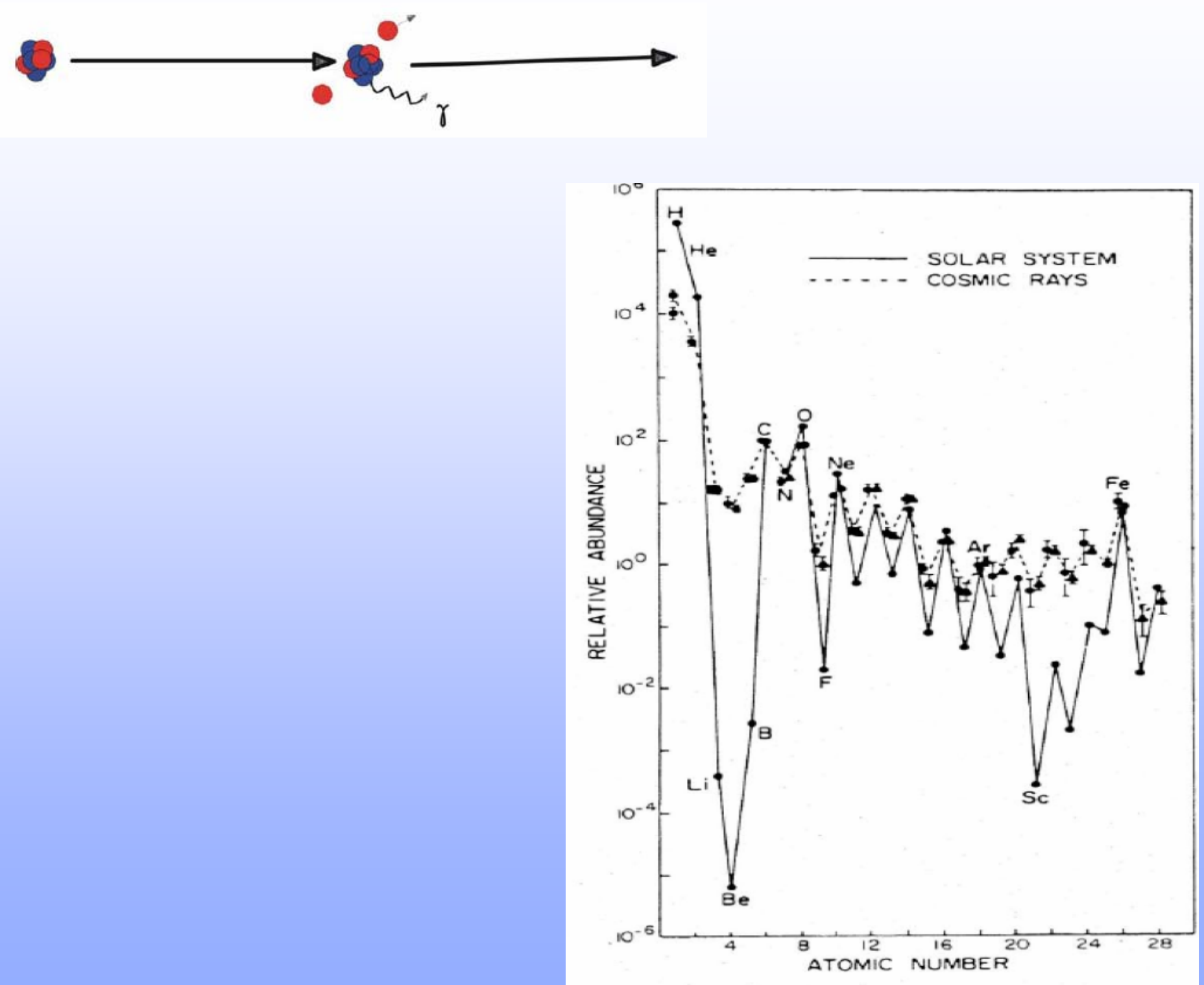


\section{Big-RIPS in RIKEN (Japan, near Tokyo)}



\section{Separation principle: $\mathrm{B} \rho-\Delta \mathrm{E}-\mathrm{B} \rho$ method}






\section{Separation principle: $\mathrm{B} \rho-\Delta \mathrm{E}-\mathrm{B} \rho$ method}



\section{The FRS at GSI}






\section{Separation and identification at the FRS}

\section{Detectors}



Principle

$B \rho=\gamma /$ * $A / Z$

ToF: $\gamma \mathrm{V}$

$\Delta \mathrm{E}: \quad \mathrm{Z}$



Identification of fragments






\section{Experimental area at the Fragment Separator FRS}






\section{Identification and experiments with few atoms per week}

\section{In-flight identification (BQ, TOF, $\triangle E$ )}

${ }^{129} \mathrm{Xe}(1095 \mathrm{AMeV})+{ }^{9} \mathrm{Be}$






\section{ISOL target and ion source}



\section{Ionisation mechanisms}

\section{Surface ionization}



hot metal surface



\section{Laser ionization}


\section{Ionization by} electron impact


ion






\section{Nuclear chart @ CERN-ISOLDE}






\section{3a. Superheavy elements}




\section{Superheavy elements}



Gottfried Münzenberg und Matthias Schädel „Moderne Alchemie - Die Jagd nach den schwersten Elementen 


\section{Chemical element 112: Copernicium (Cn)}

- Officially named in 2009 by IUPAC

- "The idea was to go backwards, to honor someone who was not greatly honored in his lifetime." - Sigurd Hofmann

- Hofmann wanted to highlight the contribution of nuclear chemistry to other fields, astrophysics in particular

- Element was first produced at GSI in 1996 by fusion of zinc and lead



\section{Synthesis and identification of SHE at SHIP}






\section{Cross section systematics (1n evaporation residue)}

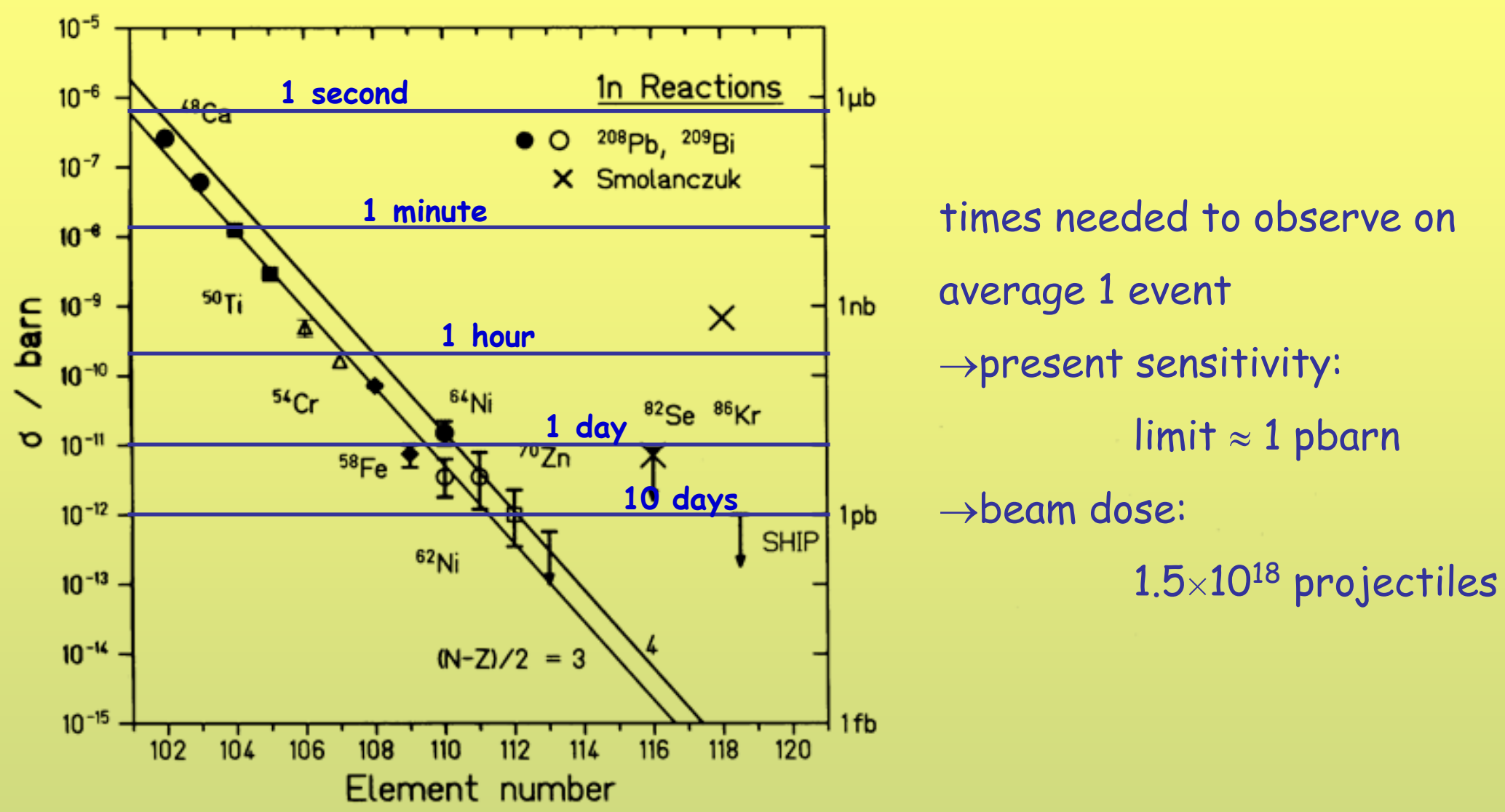




\section{Status of worldwide SHE research}

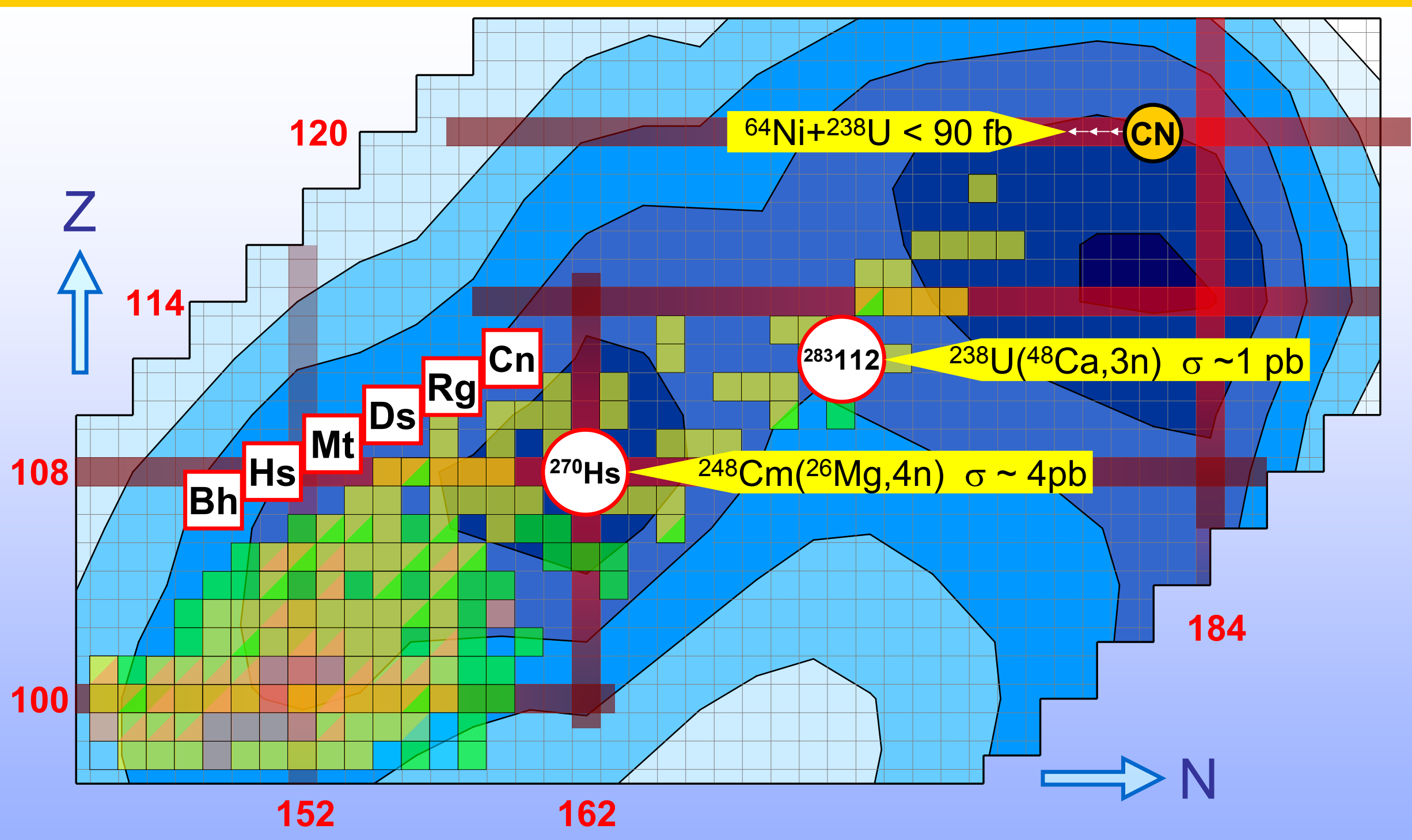

Background: calculated shell correction energies $E_{\text {shell }}$ of SHE 


\section{Atomic structure and properties of SHE}

The inner electrons move at relativistic speed in the strong electric field of the high-Z nucleus:

$\mathrm{v} / \mathrm{c} \sim \mathrm{Z} \alpha \sim 100 / 137 \rightarrow \beta \sim 0,7$

example ${ }_{106} \mathrm{Sg}$ :

$\beta=0,77$



$\gamma=1,58$

$r=0,63 r_{0}$

$\rightarrow \mathrm{s}, \mathrm{p}$-electrons are attracted closer to the nucleus

$\rightarrow$ spin-orbit splitting

$\rightarrow$ high electron-density near nuclear surface

$\rightarrow$ screening of nuc.charge for outer $(d, f)$ electrons

Chemistry of Transactinides

$\rightarrow$ electron configuration, ionic radii, binding energies

$\rightarrow$ chemical properties (redox potential, volatility, complex formation, periodicity of chem.properties,...)

Glenn Seaborg during his visit to GSI 


\section{Theory predictions: relativistic vs. non-relativistic calculations}



\section{CONSEQUENCES}

* Shift of energetic and spacial distribution of electronic orbital on an absolute and relative scale

$\rightarrow$ Change of electronic ground state configurations and the ionization energies

$\rightarrow$ Change of atomic- and ionic radii

$\rightarrow$ Change of availability of electronic orbitals for chemical bonding

$\rightarrow$ Change of bonding energies in molecular bonds

$\rightarrow$ Change of contribution of ionic- and covalent part in the bonding

\section{Group 6 elements}



$\mathrm{Sg}$

$d^{4} s^{2}$

nr. rel.

$\underline{7 s}$


V. Pershina $8 / 98$, Desclaux 


\section{Confirmation by chemistry}






\section{Hot fusion advances the field}

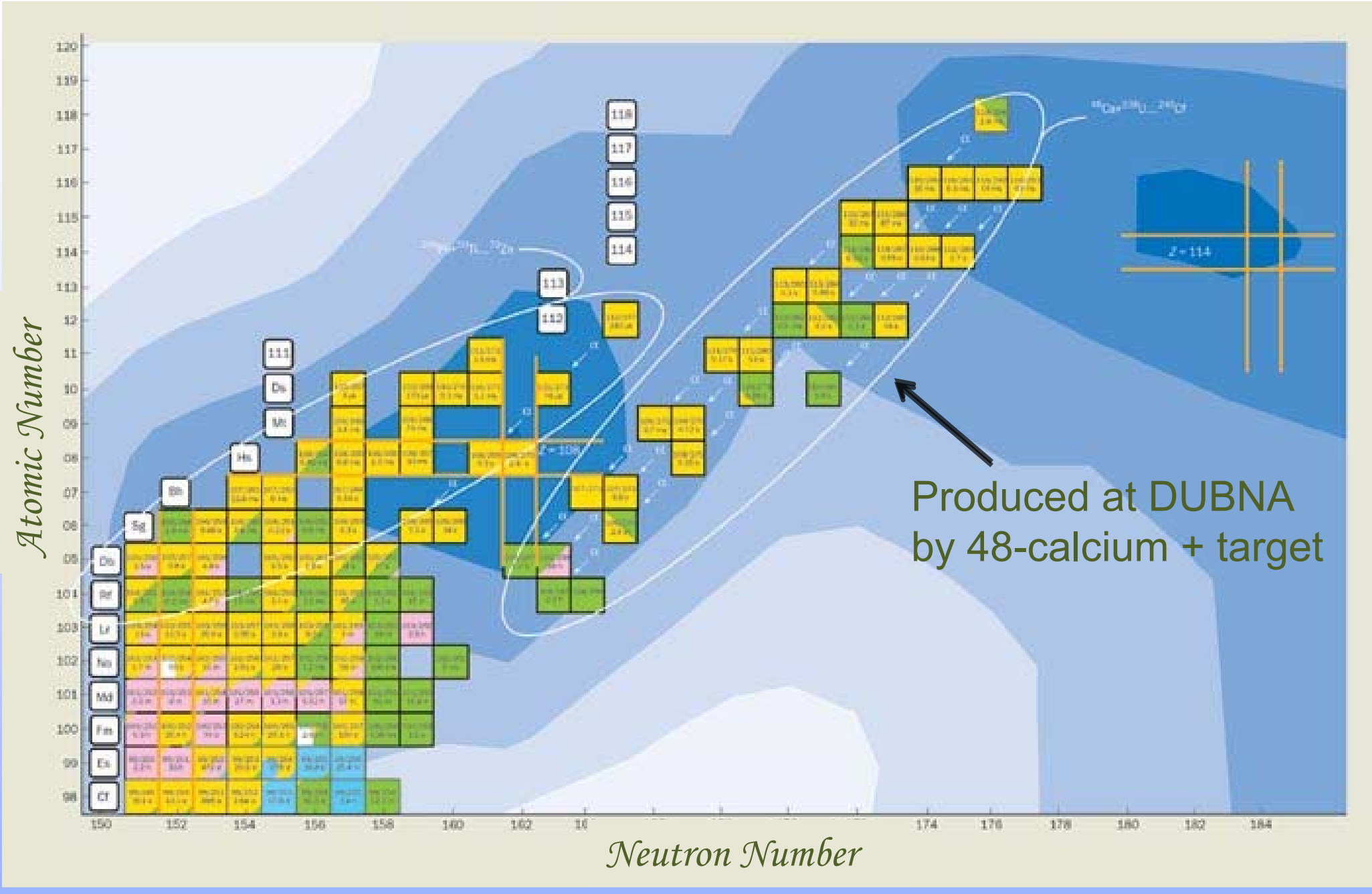




\section{3b. 2-proton radioactivity}




\section{Discovery of a new type of radioactivity}

- Production of nuclei at the proton dripline

- Study of the 2-proton emitter ${ }^{45} \mathrm{Fe}$ 


\section{Emission of two protons from nuclear states}

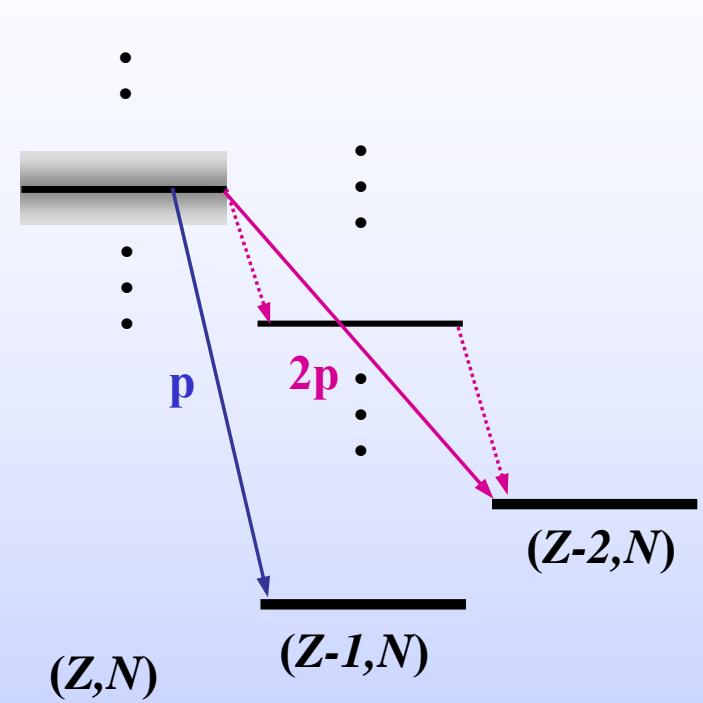

sequential

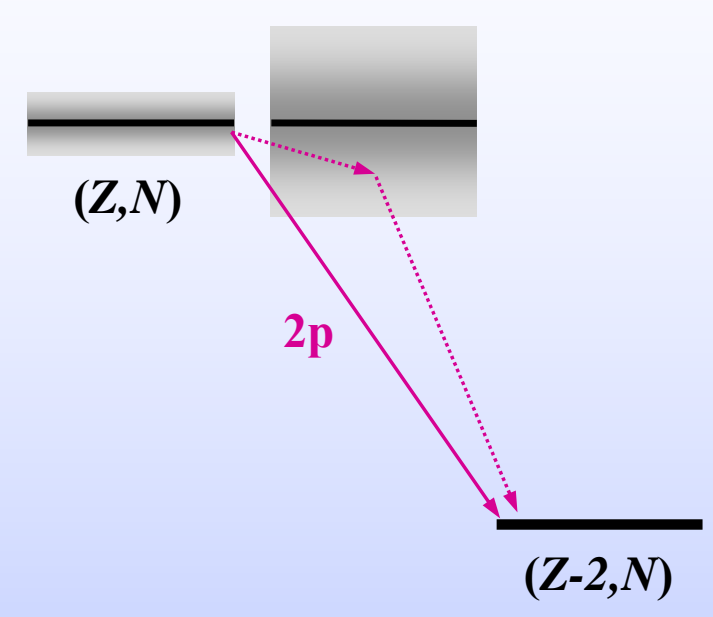

democratic

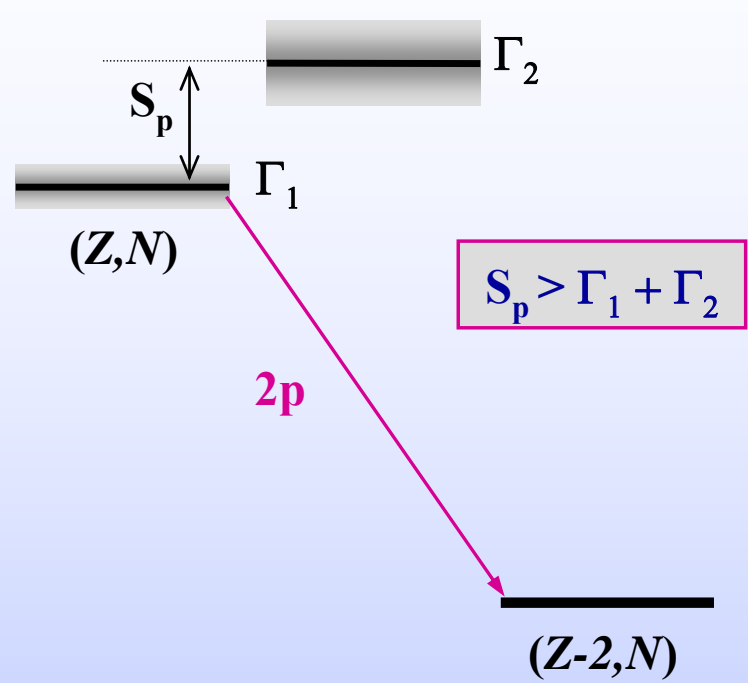

2 p radioactivity
${ }^{22} \mathrm{Mg}^{*},{ }^{26} \mathrm{Si}^{*}$ - Cable et al., 1983
${ }^{35} \mathrm{~K}^{*} \quad$ - Äystö et al., 1985
${ }^{31} \mathrm{Cl}^{*} \quad$ - Borge et al., 1990
${ }^{14} \mathrm{O}^{*}$ - Bain et al., 1996
${ }^{18} \mathrm{Ne}^{*} \quad$ - Gómez del Campo
et al., 2000

${ }^{6} \mathrm{Be}$ - Bochkarev et al., 1989

${ }^{12 O}$ - Kryger et al., 1994

${ }^{16} \mathrm{Ne}-$ ?
Not observed before !

Predicted candidates :

${ }^{19} \mathrm{Mg},{ }^{45} \mathrm{Fe},{ }^{48} \mathrm{Ni},{ }^{54} \mathrm{Zn}$ 


\section{Ground state energies of ${ }^{45} \mathrm{Fe},{ }^{44} \mathrm{Mn},{ }^{43} \mathrm{Cr}$}

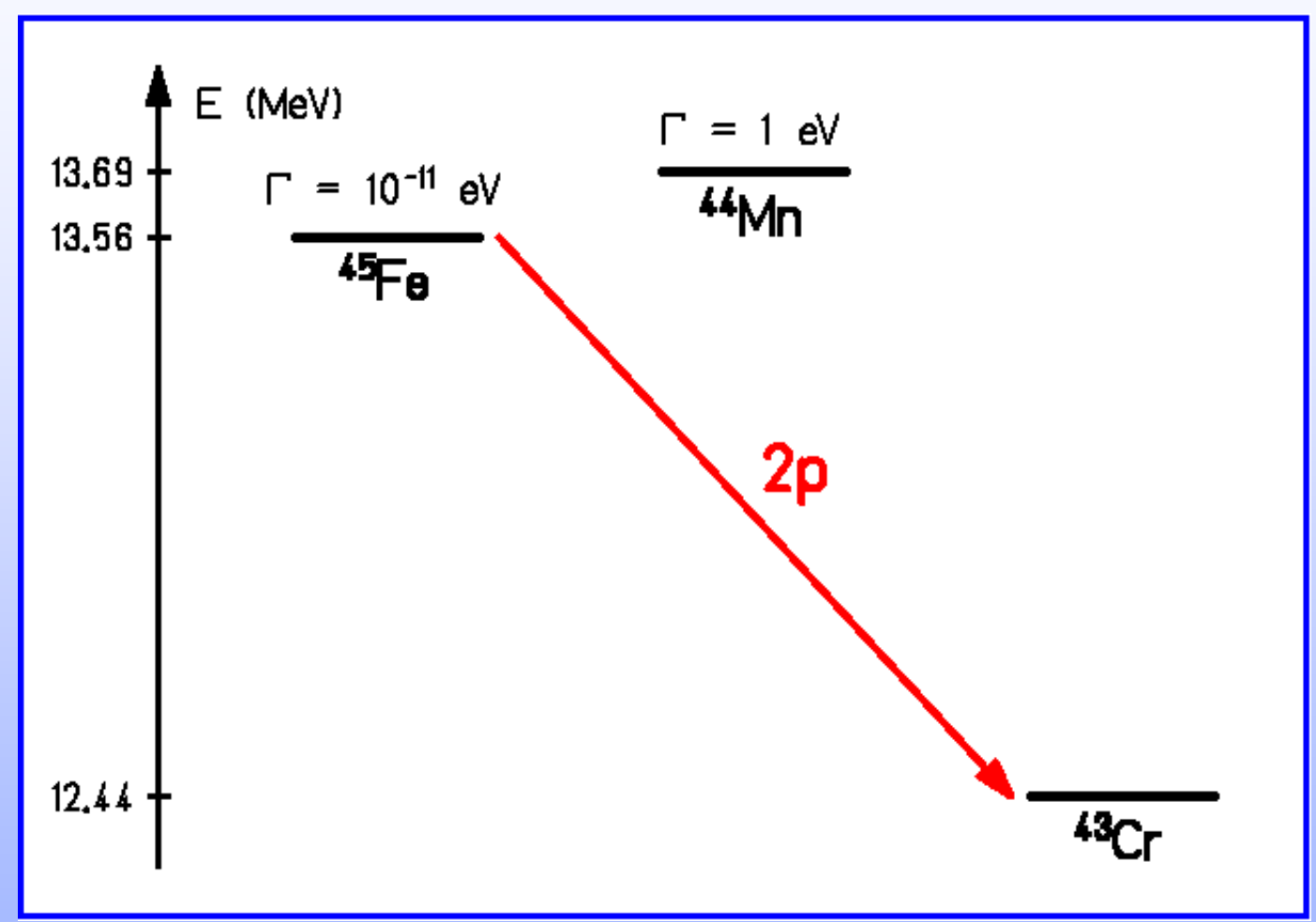

\begin{tabular}{|c|c|c|}
\hline Author & $\mathrm{Q}_{2 p}[\mathrm{MeV}]$ & $\mathrm{T}_{1 / 2}[\mu \mathrm{s}]$ \\
\hline Brown & $1.15 \pm 0.09$ & $2-300$ \\
\hline Ormand & $1.28 \pm 0.18$ & $0.01-100$ \\
\hline Cole & $1.22 \pm 0.05$ & - \\
\hline
\end{tabular}

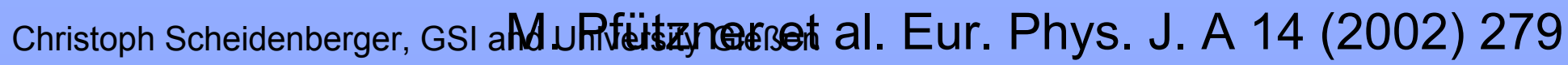




\section{Experiment at the FRS}



M. Pfützner et al. Eur. Phys. J. A14 (2002) 279 


\section{Identification of ${ }^{45} \mathrm{Fe}$}

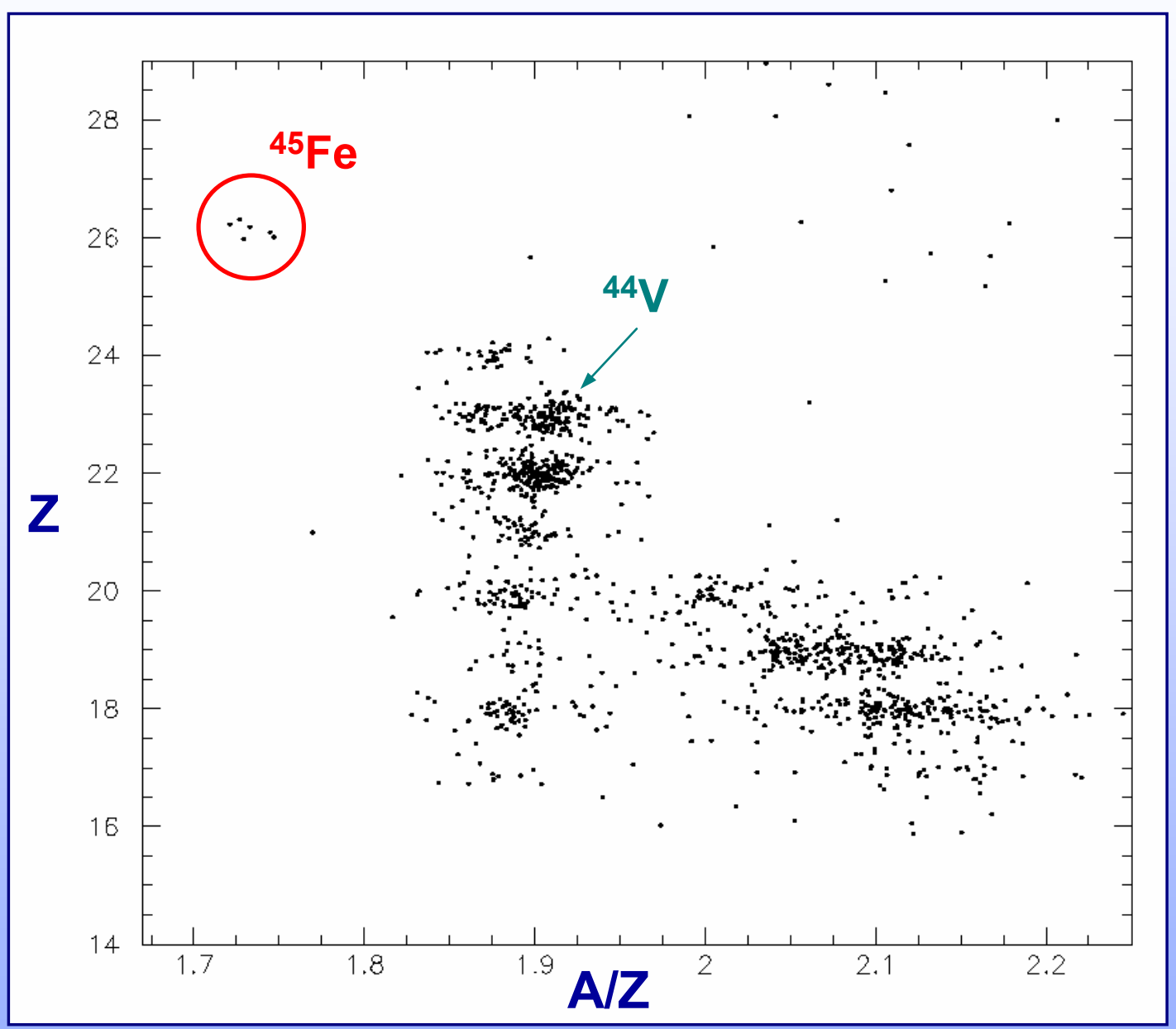

Christoph Scheidenberger, GSI and University Gießen
2115 events in 8117 min. (5.6 d)

6 events ${ }^{45} \mathrm{Fe}$

M. Pfützner et al.,

Eur. Phys. J. A 14 (2002) 279 


\section{The ${ }^{45} \mathrm{Fe}$ experiment at GANIL}

\section{LISE 3 Separator at GANIL}




Decay energies

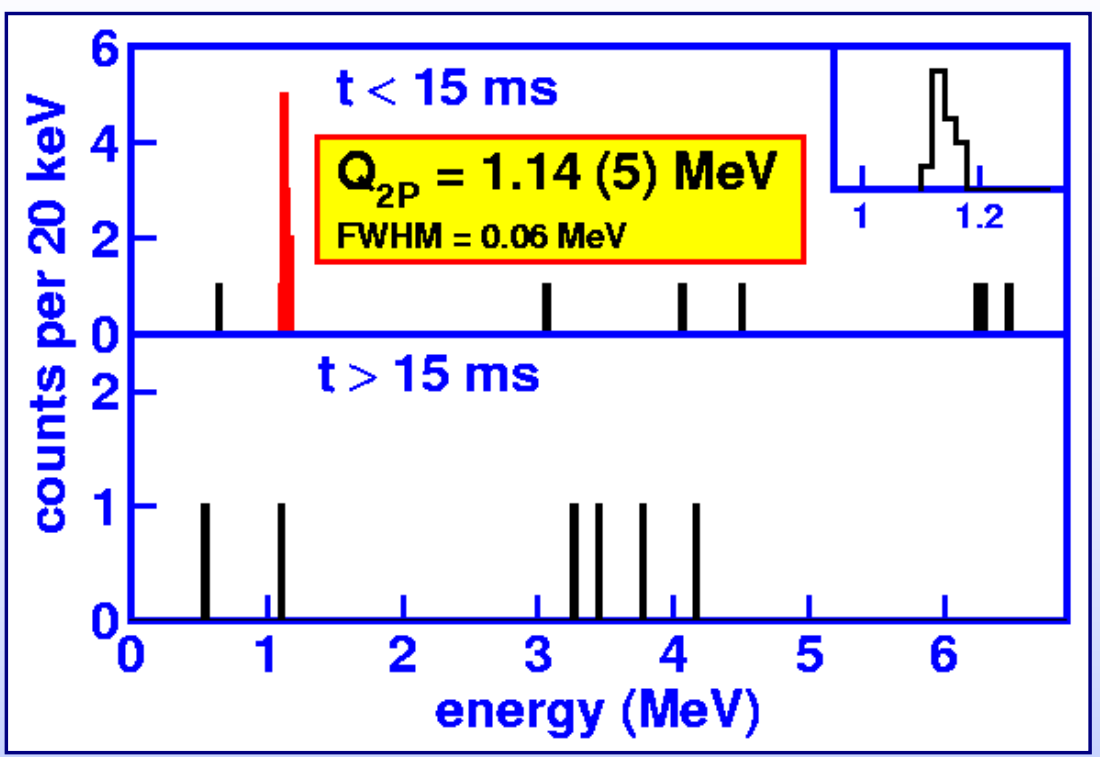

Decay times

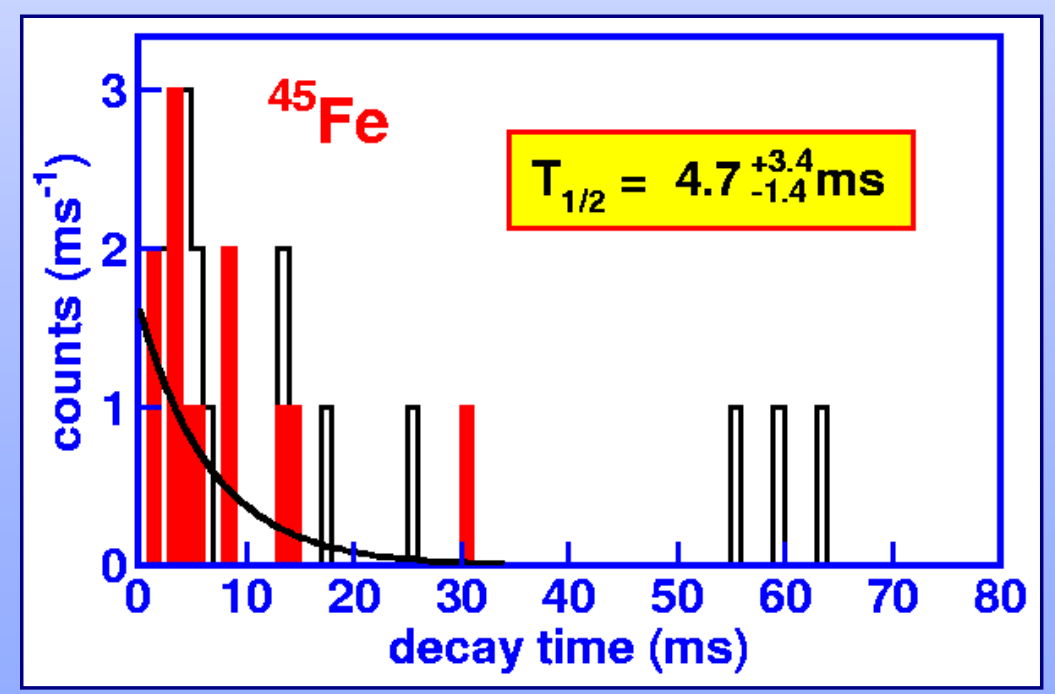




\section{$2 p$-decay of ${ }^{45} \mathrm{Fe}$ in a 3 -body model}

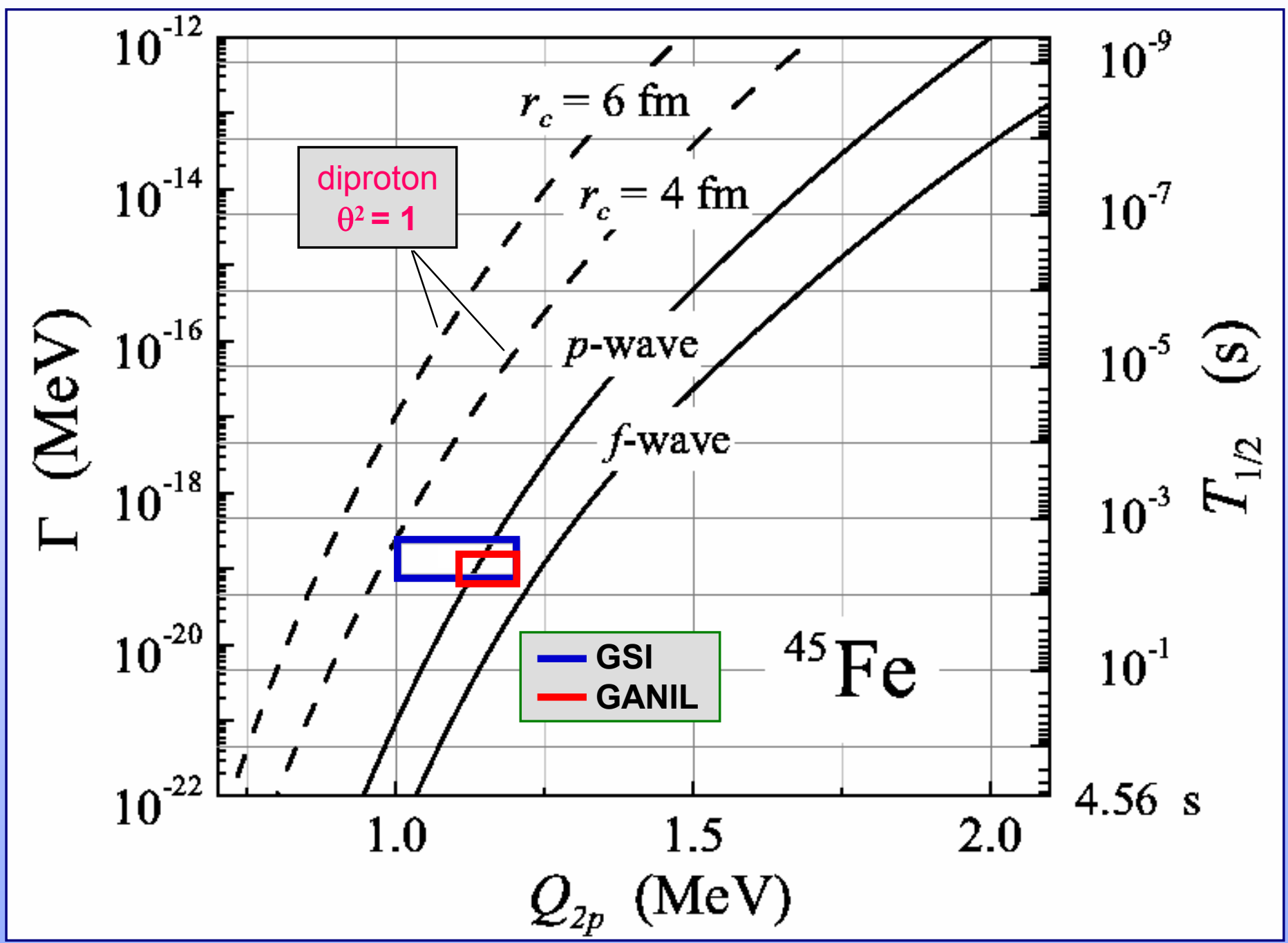




\section{3c. Halo nuclei}




\section{Halo nuclei}

- Radii measurements

- Momentum measurements

- Complete kinematic measurements 


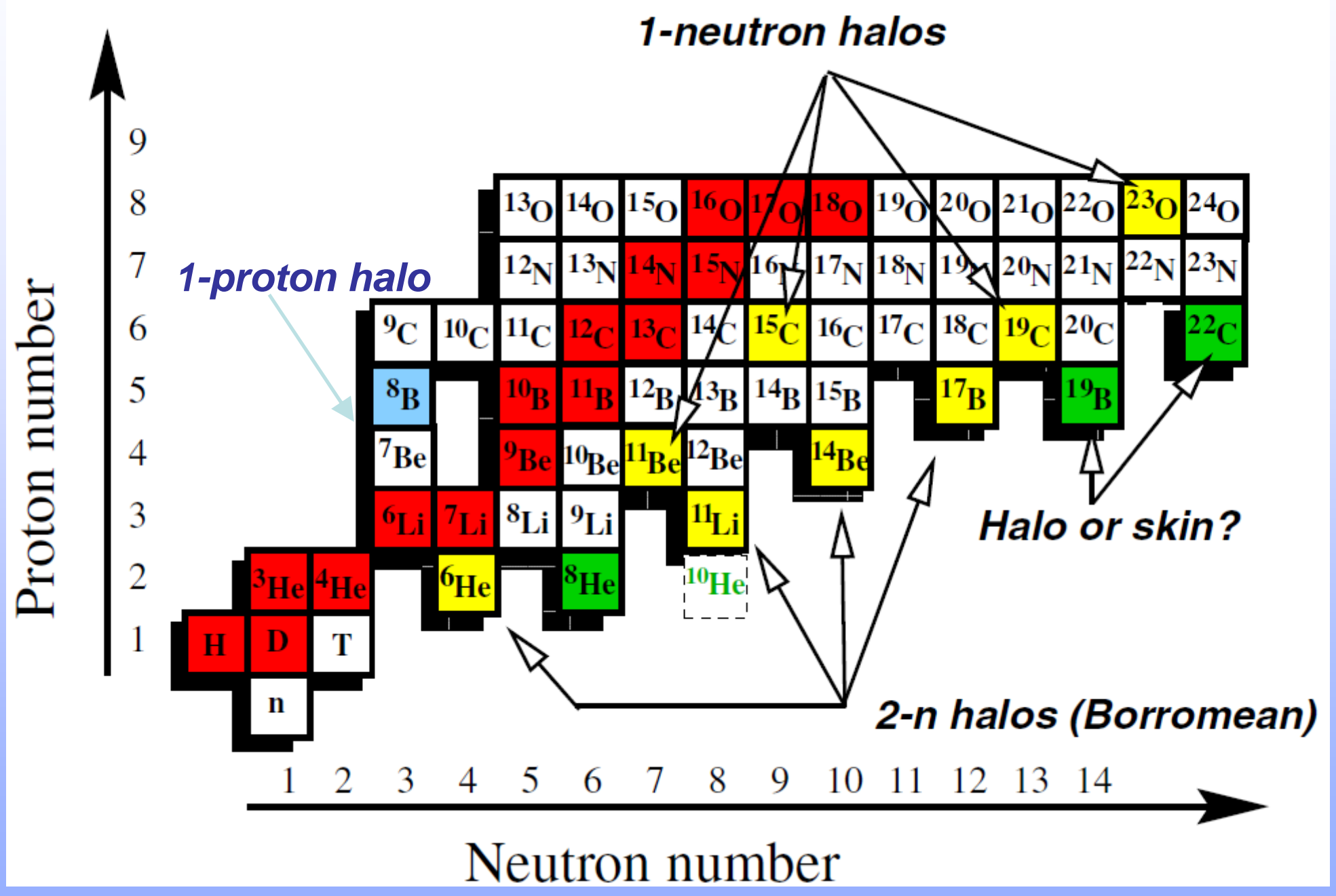




\section{Examples and simple imagination}
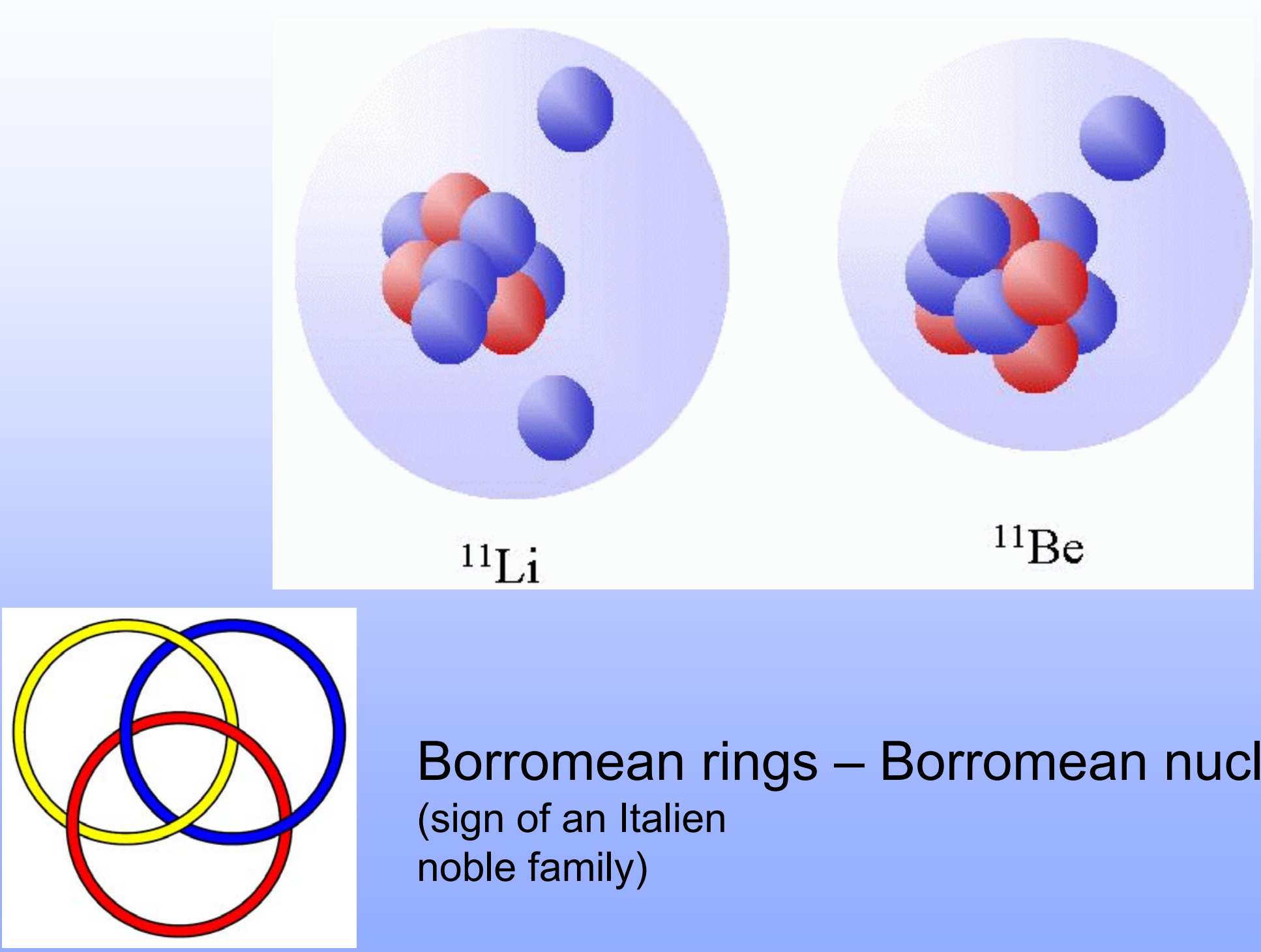

Borromean rings - Borromean nuclei (sign of an Italien noble family) 

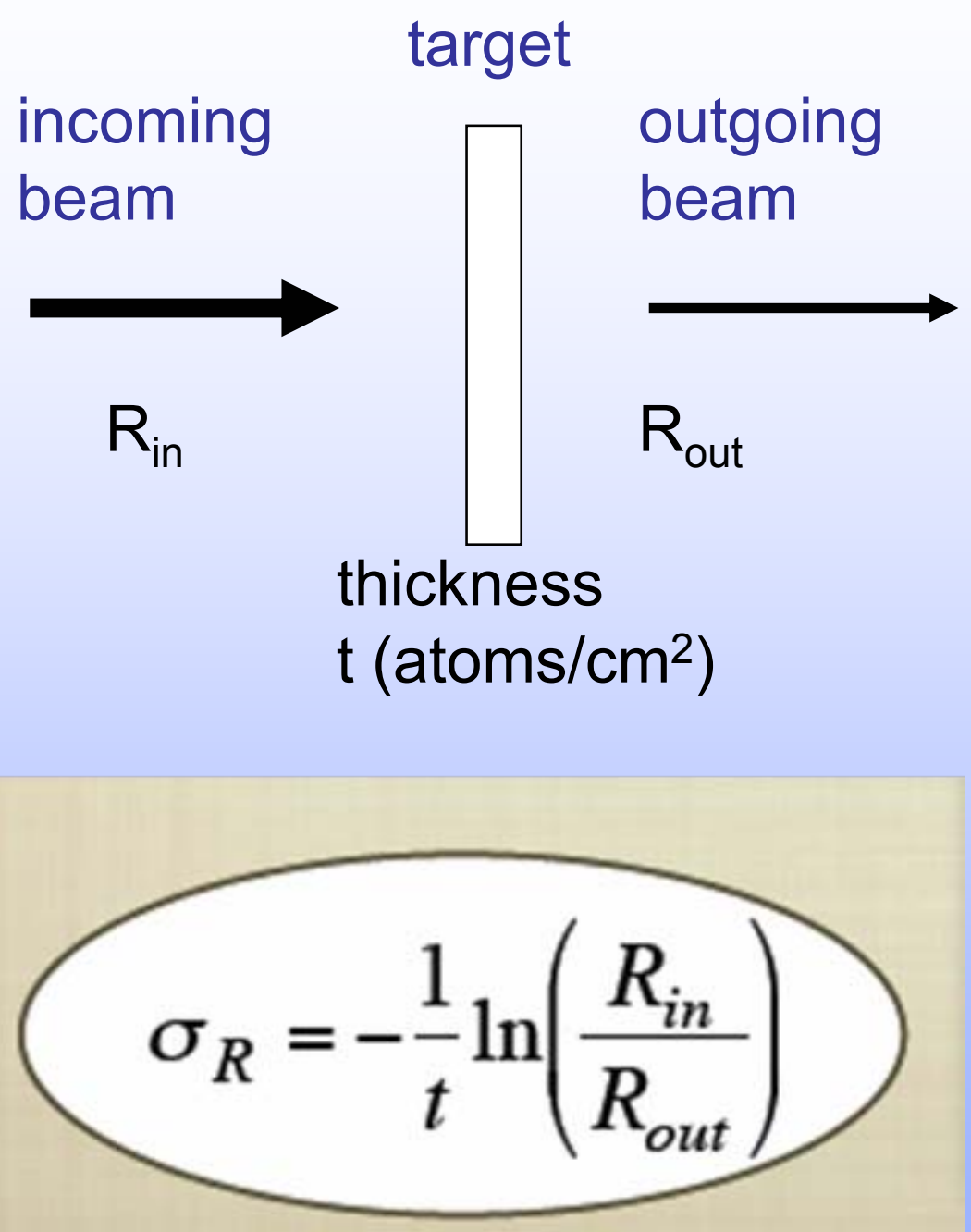

I. Tanihata et al., PRL 55 (1985) 2676 


\section{Interaction cross sections of n-rich nuclei}

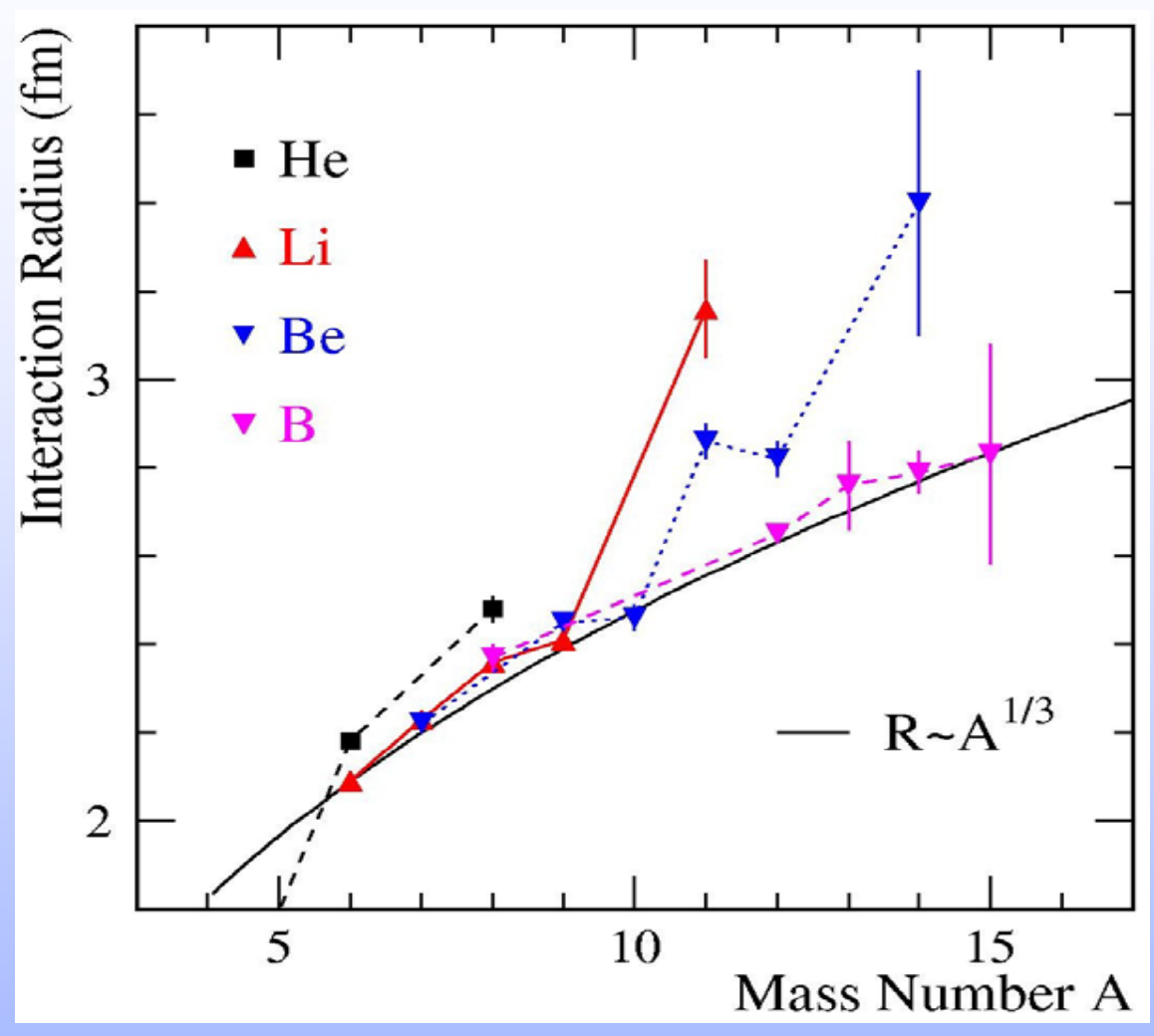

I. Tanihata et al.,

PRL 55 (1985) 2676, PLB 206 (1988) 592

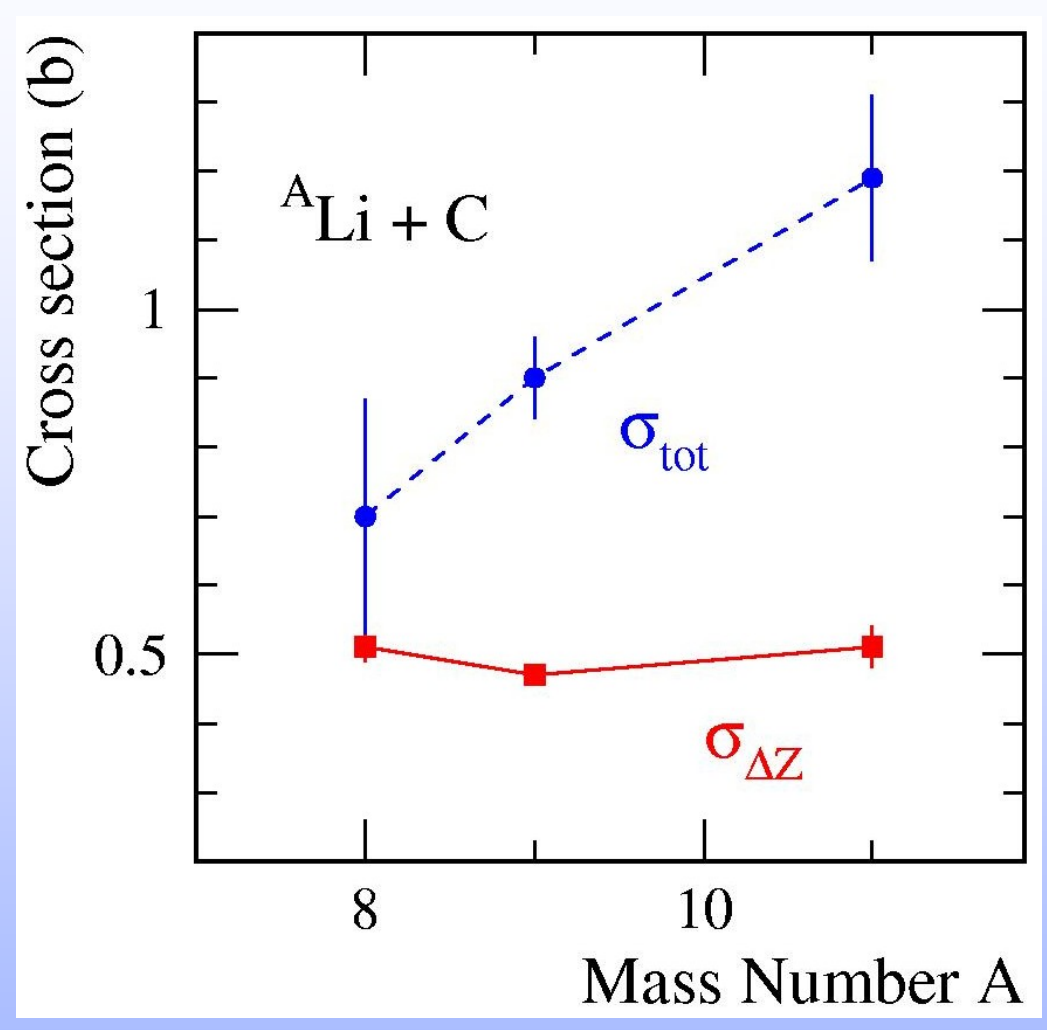

B. Blank et al.,

Z. Phys. A 343 (1992) 375c 


\section{Radial density distributions}

\section{ground state densities :}

\section{g.s. densities $\times \mathbf{r}^{2}$ :}

Theory: H. Lenske
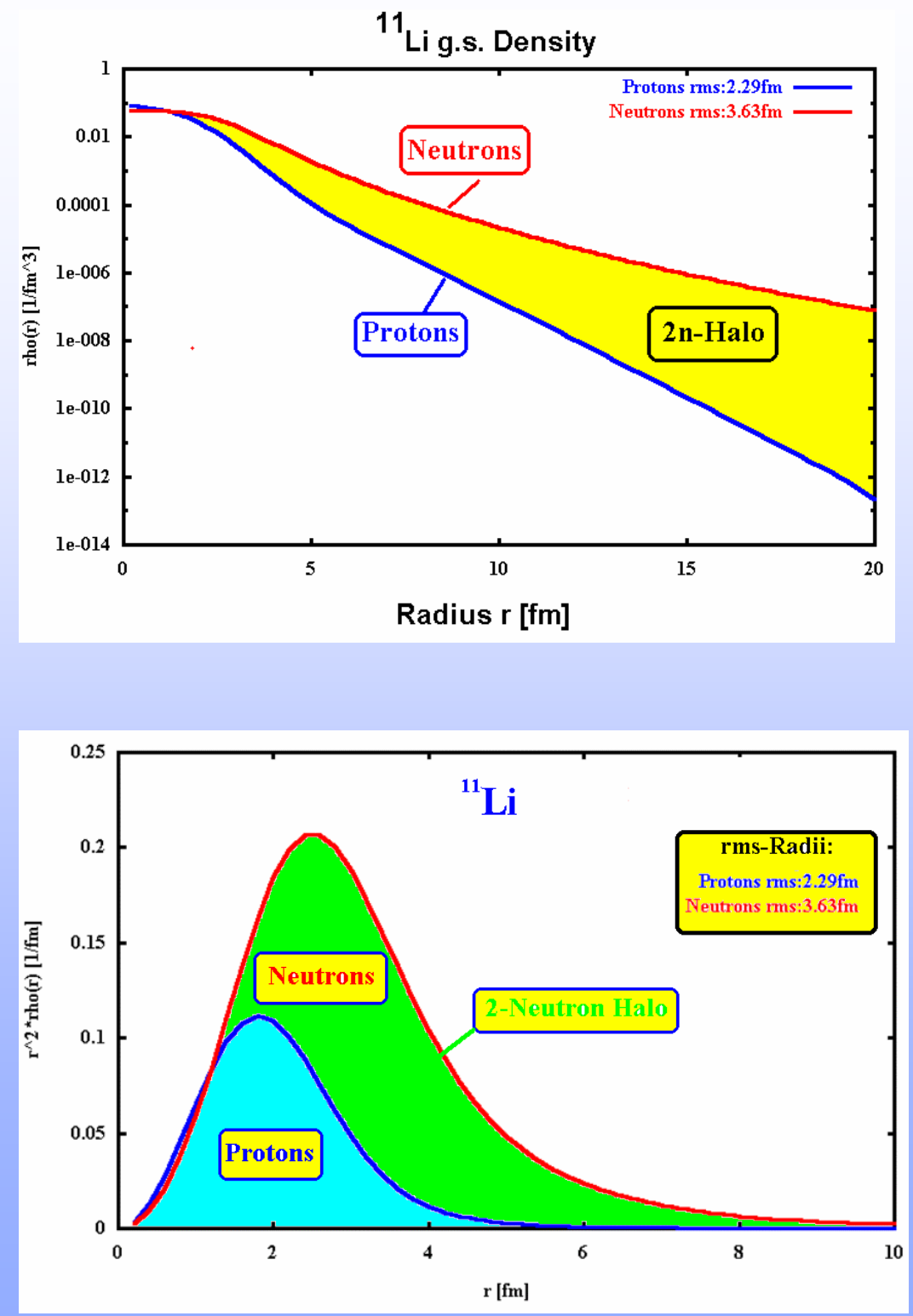


\section{Spectroscopy by one-nucleon knockout reactions}

\section{Projectile}

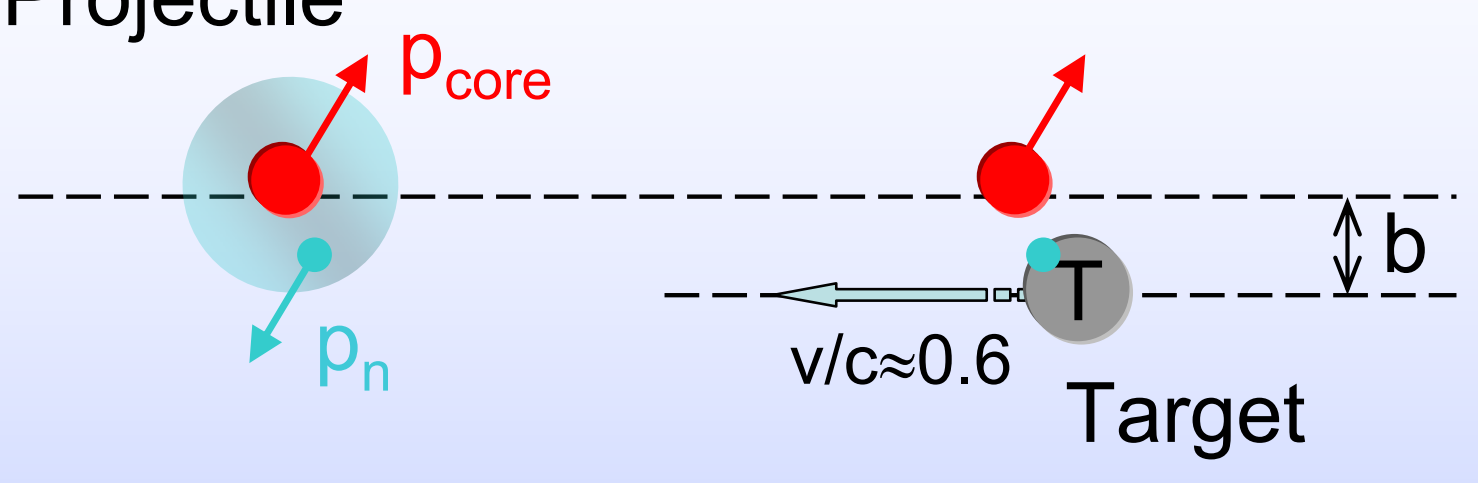

"Sudden collision": $\Delta \mathrm{t}_{\text {collision }} \ll \Delta \mathrm{t}_{\text {orbit }}$

Reaction time

$\Delta \mathrm{t}_{\text {collision }} \approx 10^{-22} \mathrm{~s}$

Internal motion

$$
\Delta \mathrm{t}_{\text {orbit }} \approx 10^{-21} \mathrm{~s}
$$

$$
\Rightarrow \mathrm{p}_{\text {core }}=-\mathrm{p}_{\mathrm{n}}
$$

$\Rightarrow$ Measurement of momentum of halo-nucleon 


\section{Transformation of wave function to momentum space}

Relation of space and momentum is given by Heisenberg's uncertainty principle:

$$
\Delta \mathrm{p} \cdot \Delta \mathrm{x} \approx \hbar
$$

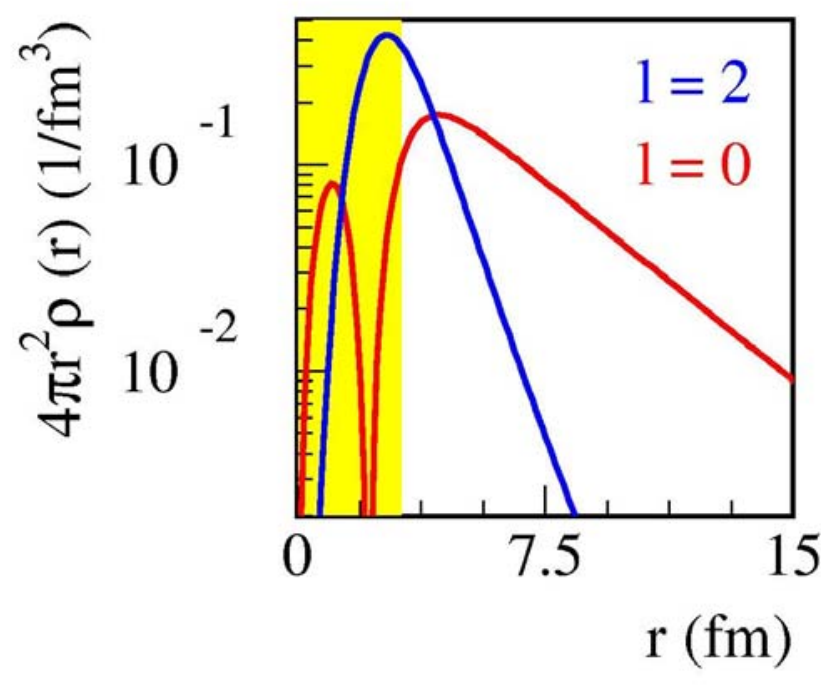

Extended

Wavefunction

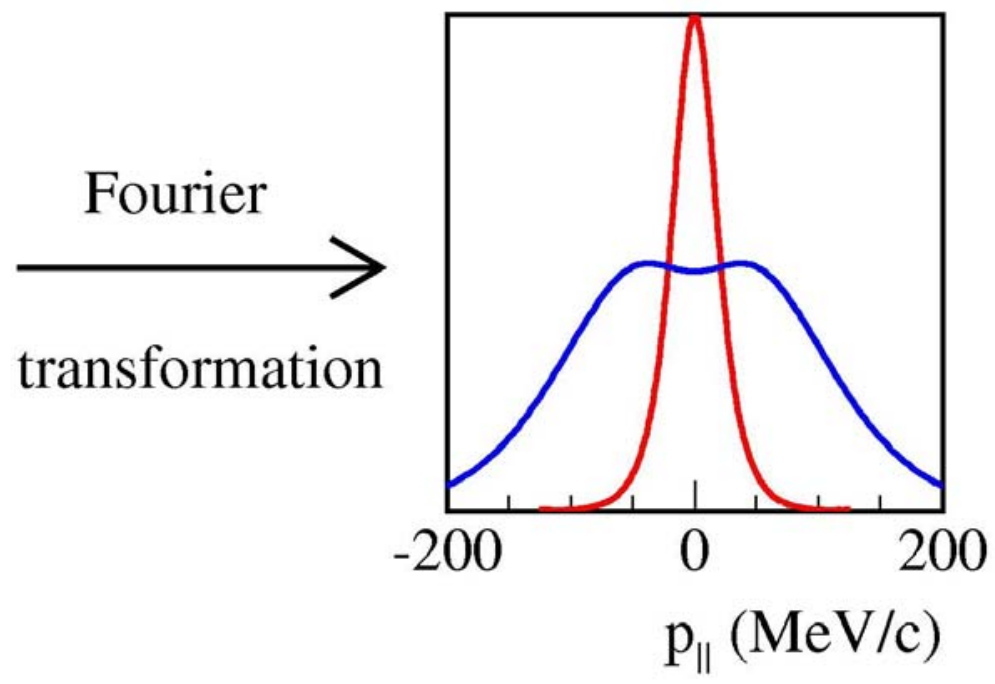

Narrow

Momentum Distribution 


\section{Momentum distributions of carbon isotopes}

Example: Carbon isotopes

$$
{ }^{A} \mathrm{C}+\mathrm{C} \rightarrow{ }^{\mathrm{A}-1} \mathrm{C}+\mathrm{x}
$$

$\mathrm{E} \approx 900 \mathrm{MeV} / \mathrm{u}$

FRS@GSI

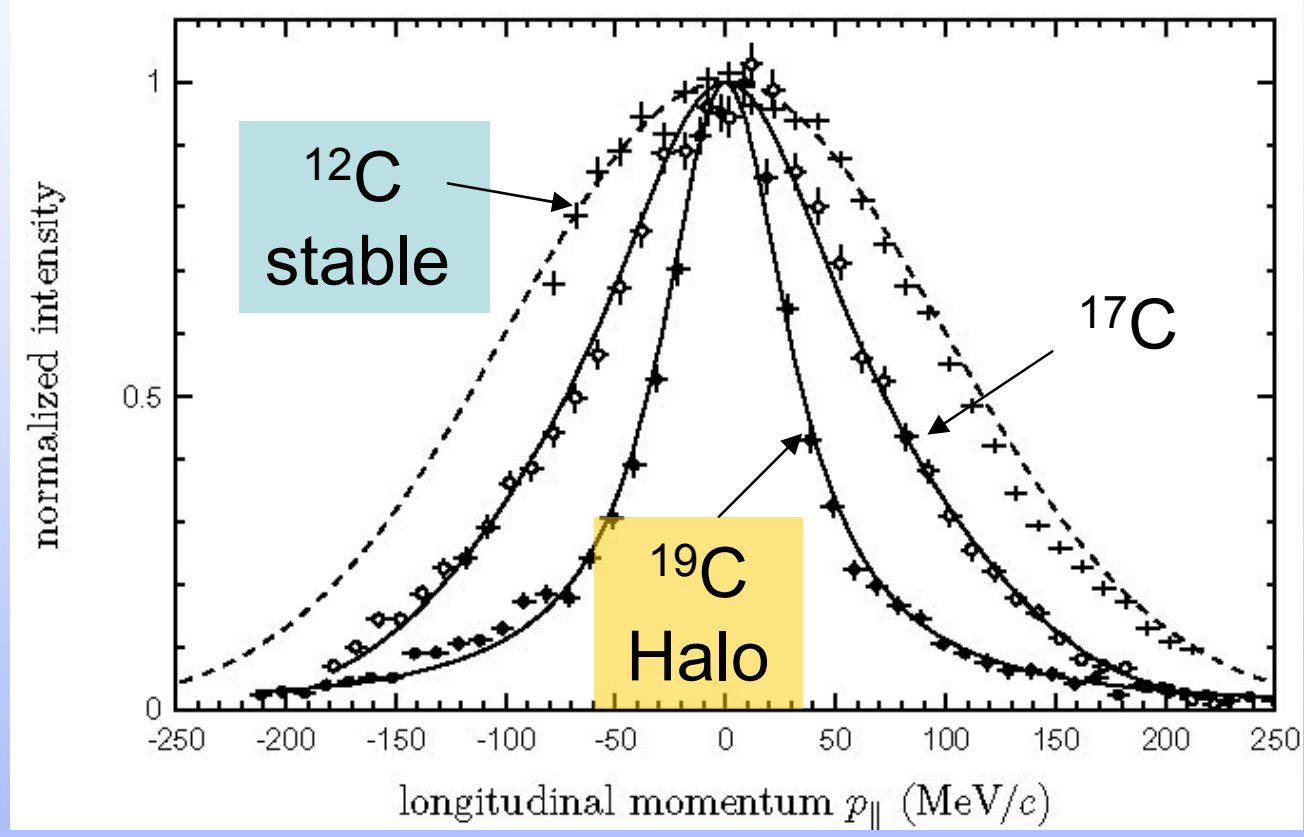




\section{High resolution momentum measurements - proton-halo in ${ }^{8} \mathrm{~B}$}

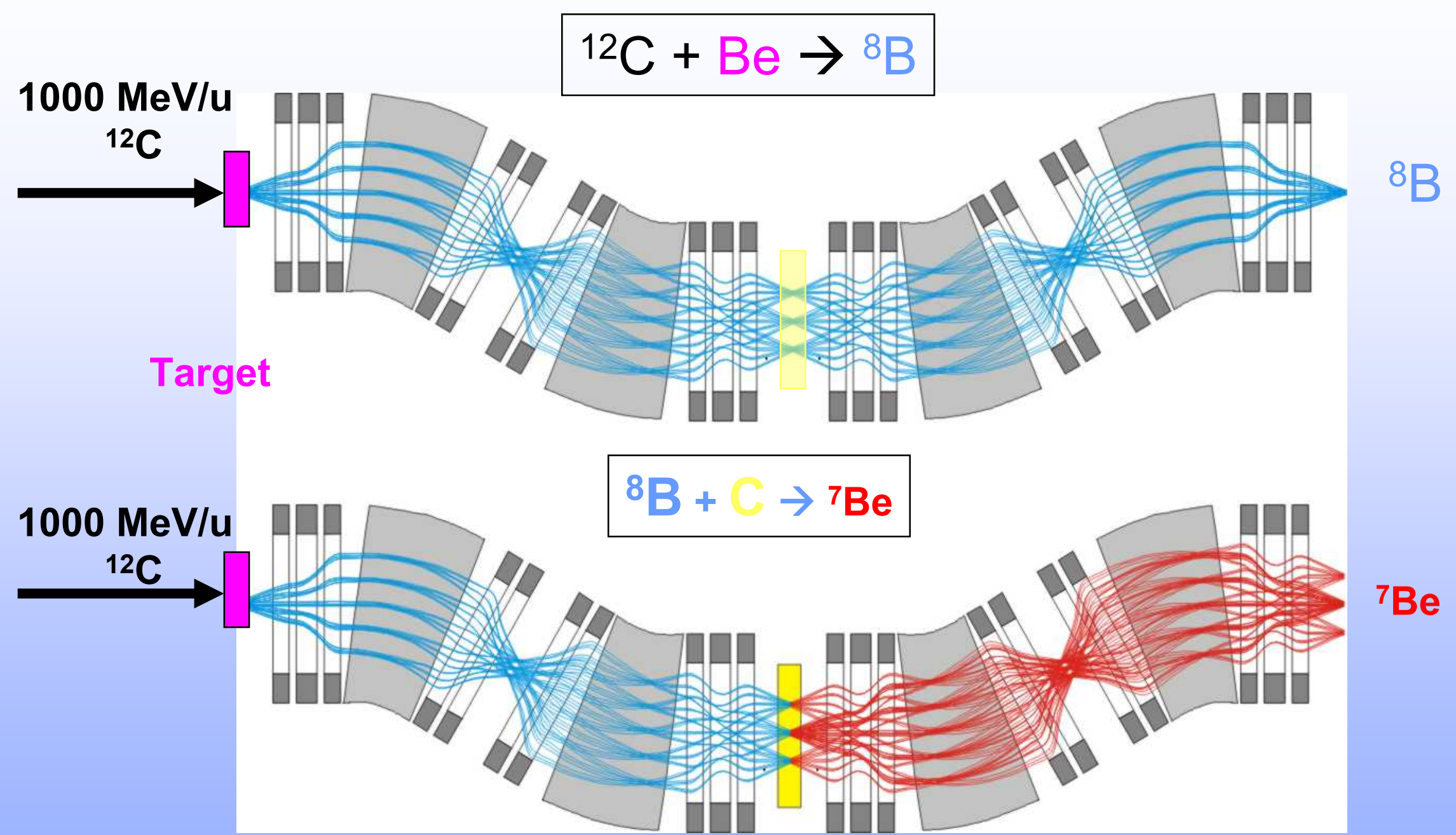

W. Schwab et al., Z. Phys. A350 (1995) 283 


\section{Discovery of a proton-halo nucleus: ${ }^{8} \mathrm{~B}$}

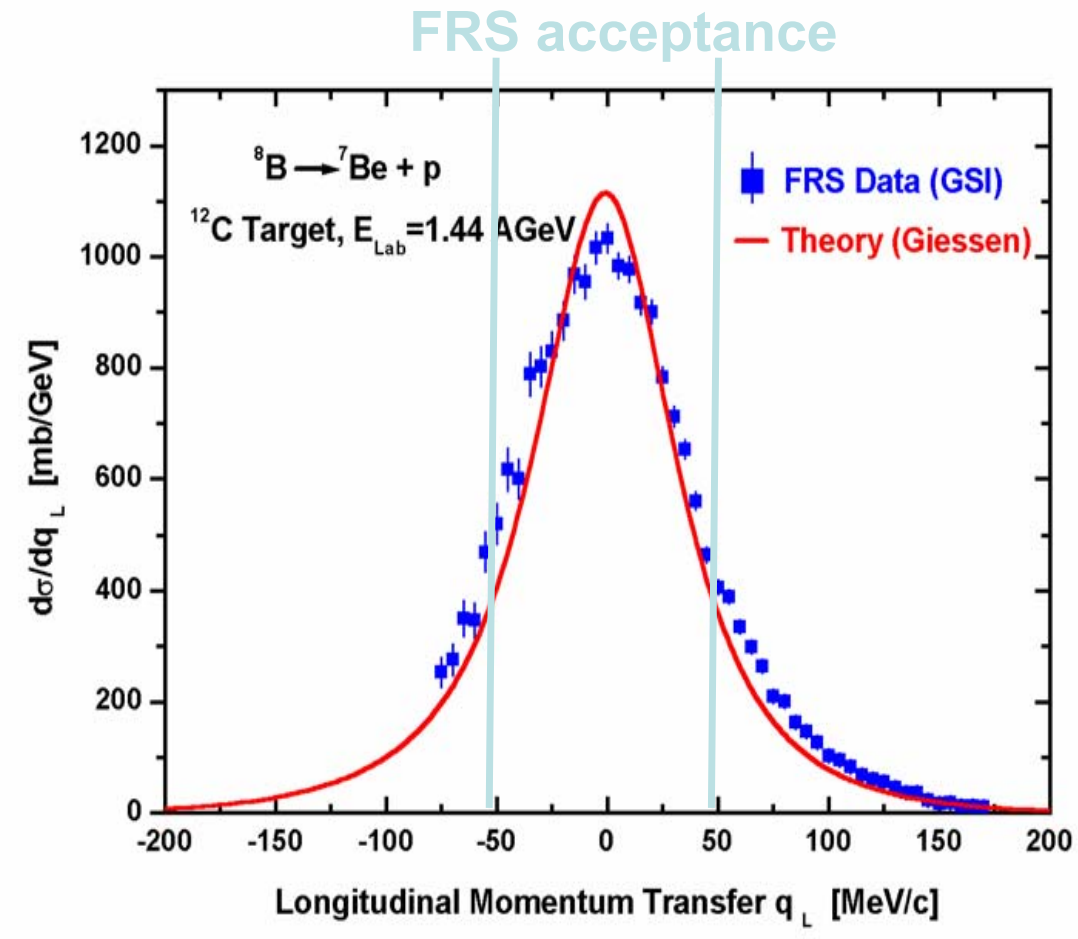

\section{$1,4 \mathrm{GeV} / \mathrm{u}^{8} \mathrm{~B} \rightarrow \mathrm{C}$}

Mean-Field \& RPA

${ }^{7} \mathrm{Be}(3 / 2-, 0.0 \quad$ ) p3/2: 71\%

${ }^{7} \mathrm{Be}(3 / 2-, 0.0 \quad) \mathrm{p} 1 / 2: 13 \%$

${ }^{7} \mathrm{Be}(3 / 2-, 0.0$ ) $\mathrm{f} 7 / 2: 11 \%$ ${ }^{7} \mathrm{Be}(3 / 2-, 0.0 \quad$ ) f $5 / 2: 5 \%$ ${ }^{7} \mathrm{Be}(1 / 2-, 0.420) \mathrm{p} 3 / 2: 15 \%$

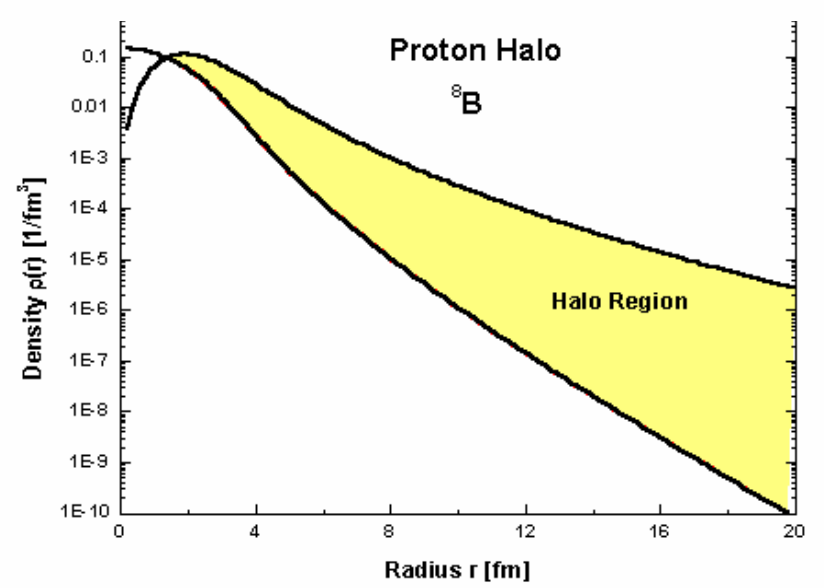

W. Schwab et al., Z. Phys. A350 (1995) 283

H. Lenske et al., Prog. Part. Nucl. Phys. 46 (2001) 


\section{Small nucleon separation energy $\rightarrow$ close to drip-lines}

Low orbital angular momentum $(\mathrm{l}=0,2)$

Asymptotic form of wave function:

$$
\Psi(\mathrm{r}) \sim \exp \left[-\left(2 \cdot \mu \cdot S_{2 n}\right)^{1 / 2} \cdot r / \hbar\right]
$$

see P. G. Hansen and B. Jonson, Europhys. Lett. 4, 409 (1987)

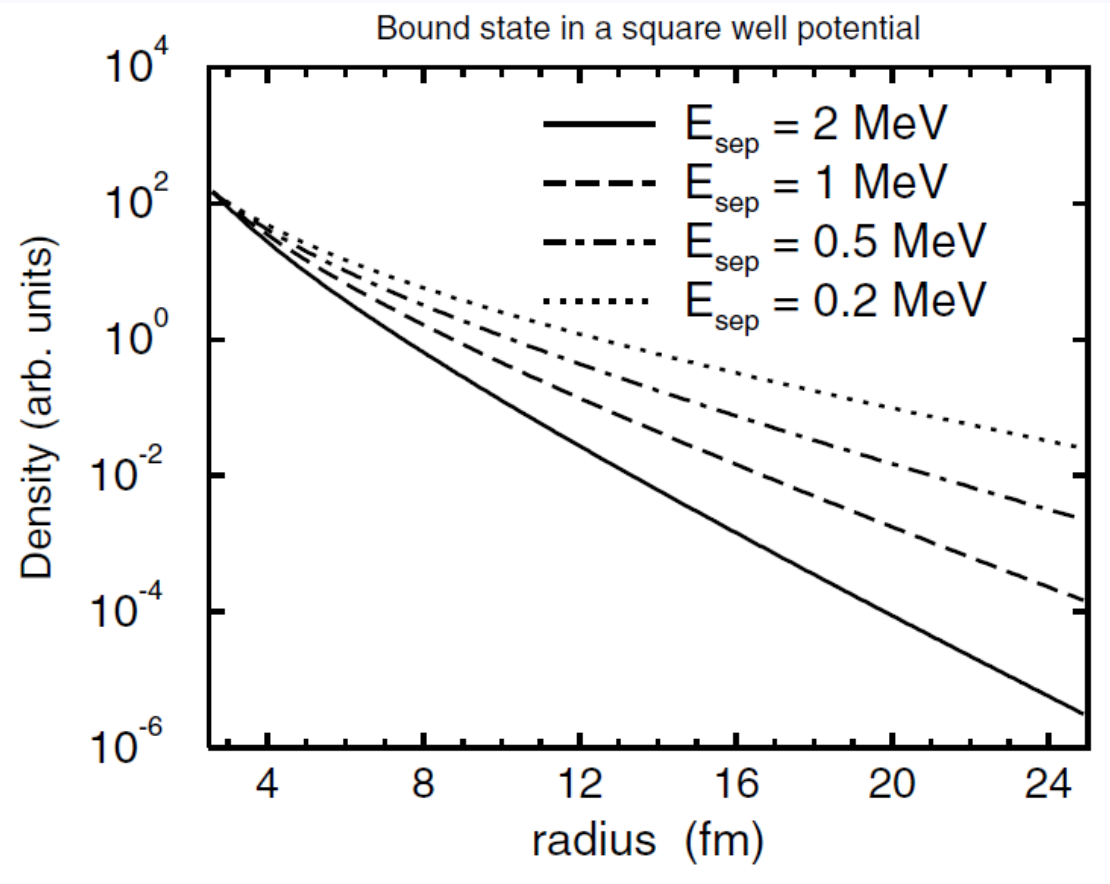

Fig. 4. The dependence of the wavefunction tail of a particle bound inside a square well potential on separation energy (the distance from the top of the well). 


\section{Kinematical complete experiments}

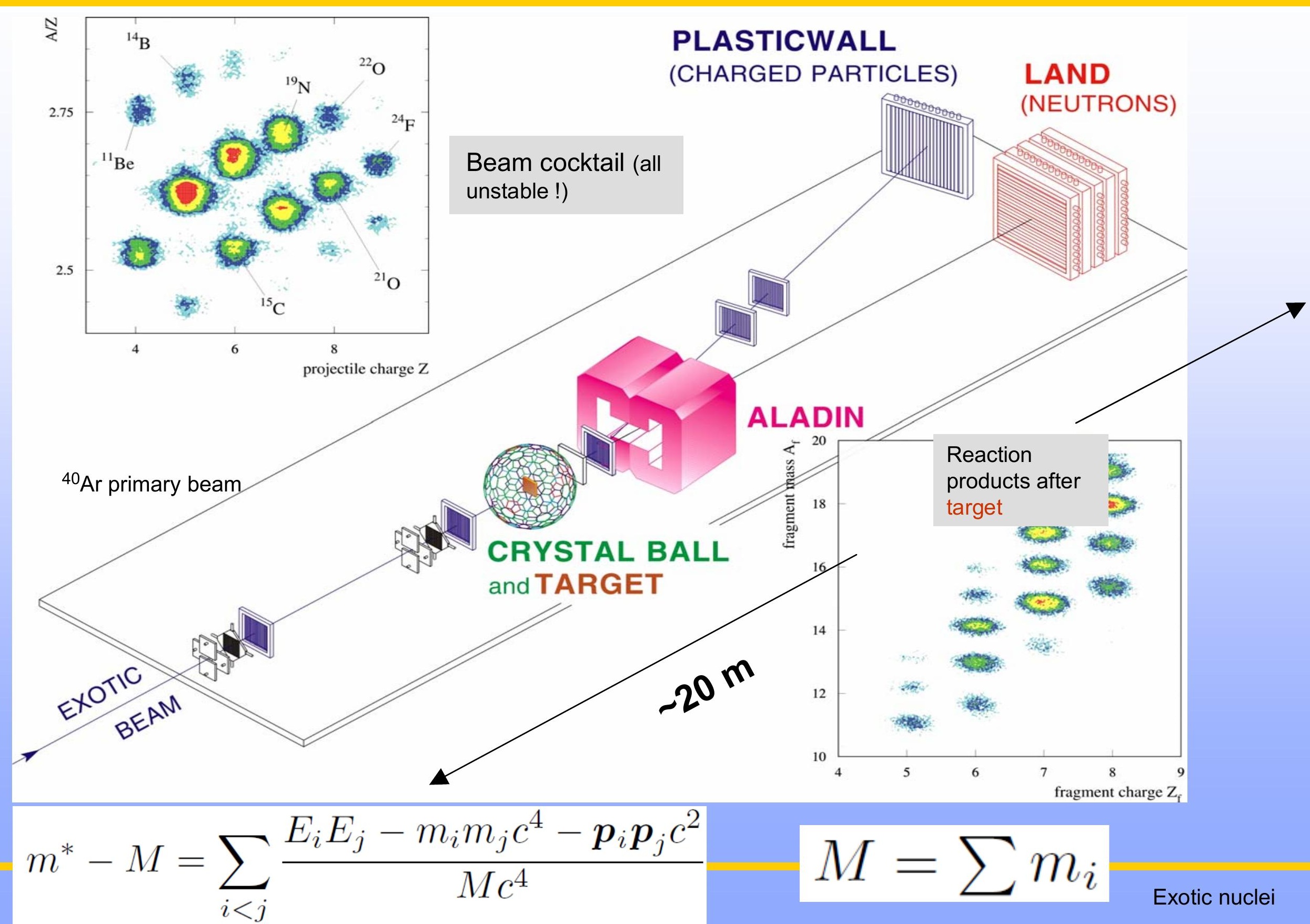




\section{Angular correlations reveal inner structure}
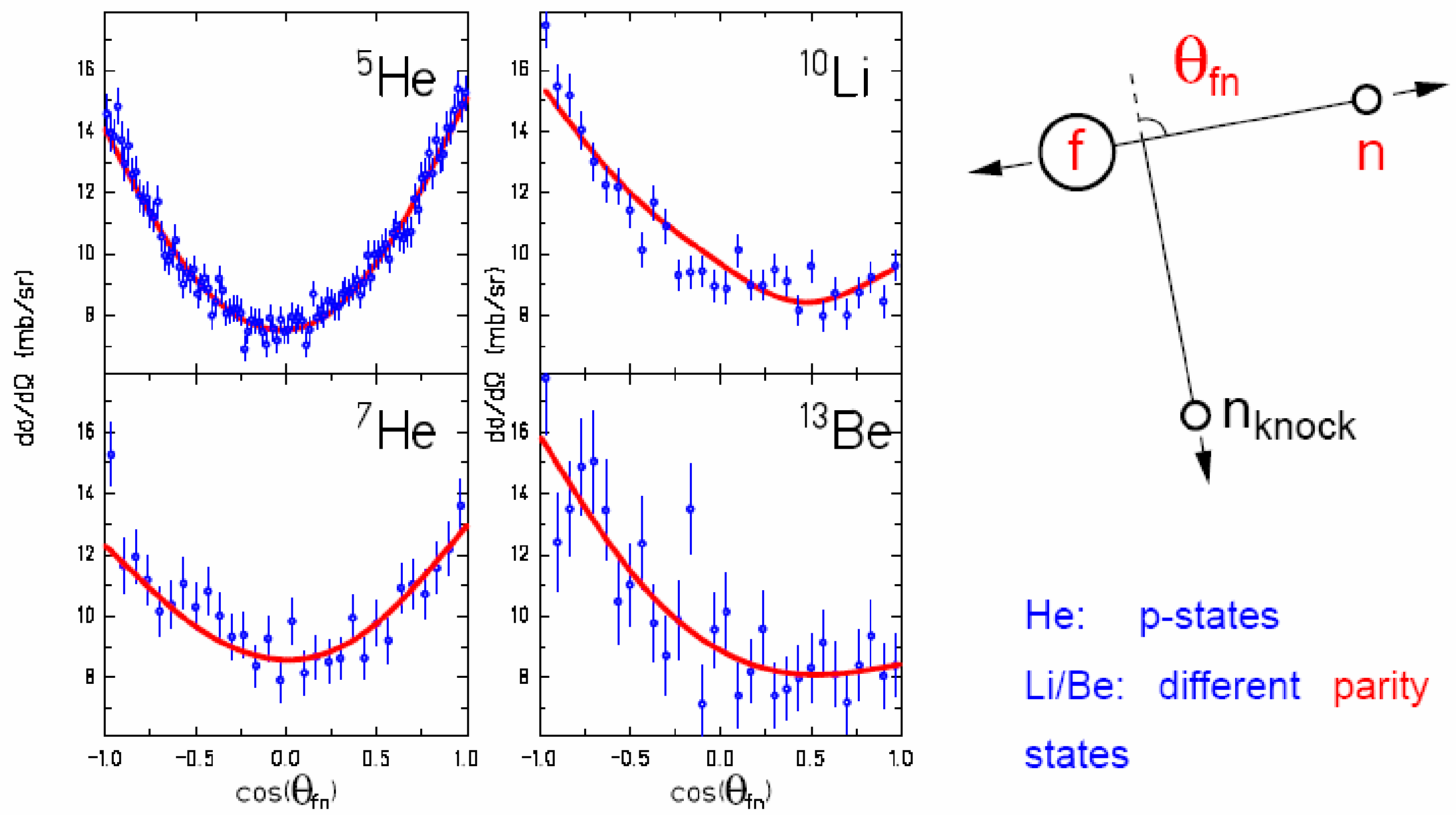

He: p-states

Li/Be: different parity states 


\section{Energy and angular correlations}

$5 \mathrm{H}$ obtained in proton knockout:

${ }^{6} \mathrm{He} \rightarrow \mathrm{p}+{ }^{3} \mathrm{H}+\mathrm{n}+\mathrm{n}$

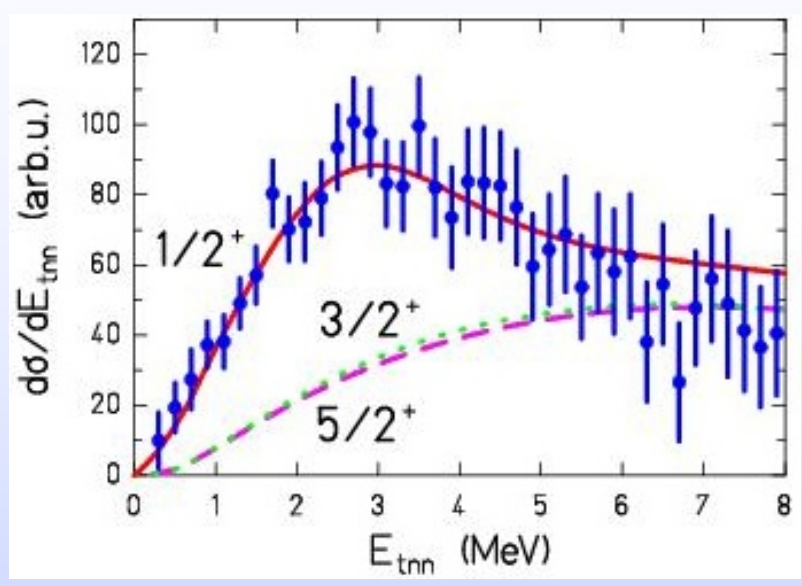

Measured correlations are consistent with a 3body microscopic calculation assuming $\mathrm{J}^{\pi}=1 / 2^{+}$

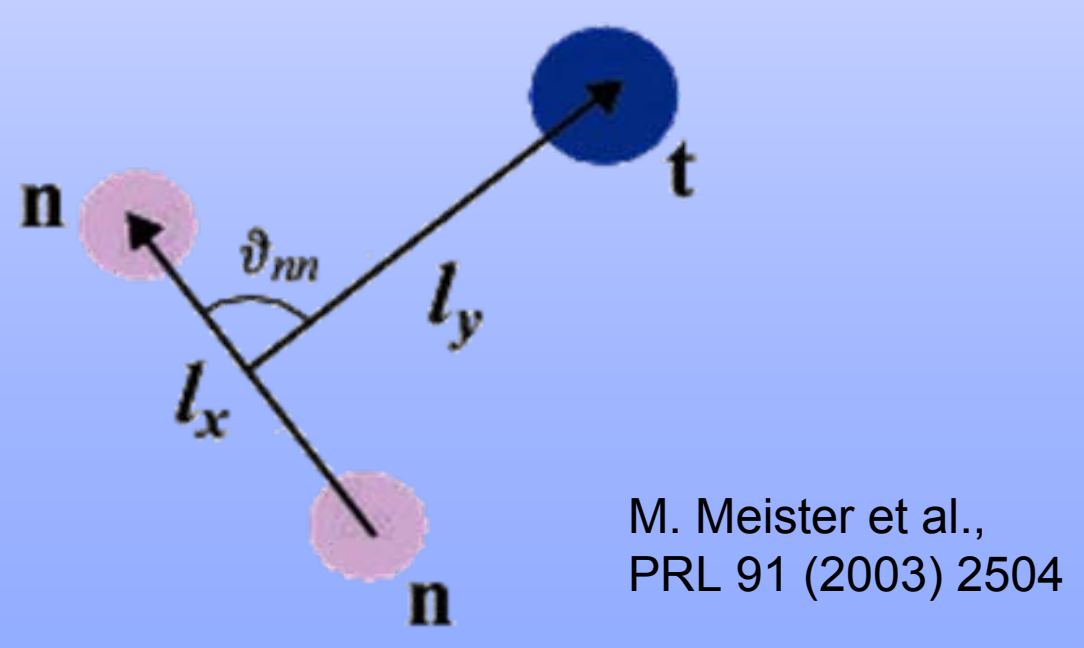

Measured three-body correlations (projections of energy and angle) are analyzed via a Jacobi coordinate system and an expansion with a restricted set of hyperspherical harmonics:

$\rightarrow$ angle between relative momenta $\theta_{n n}$

$\rightarrow$ energy sharing $\varepsilon=E_{n n} / E_{\text {total }}$ between sub-systems

$\rightarrow$ spin and parity of the state

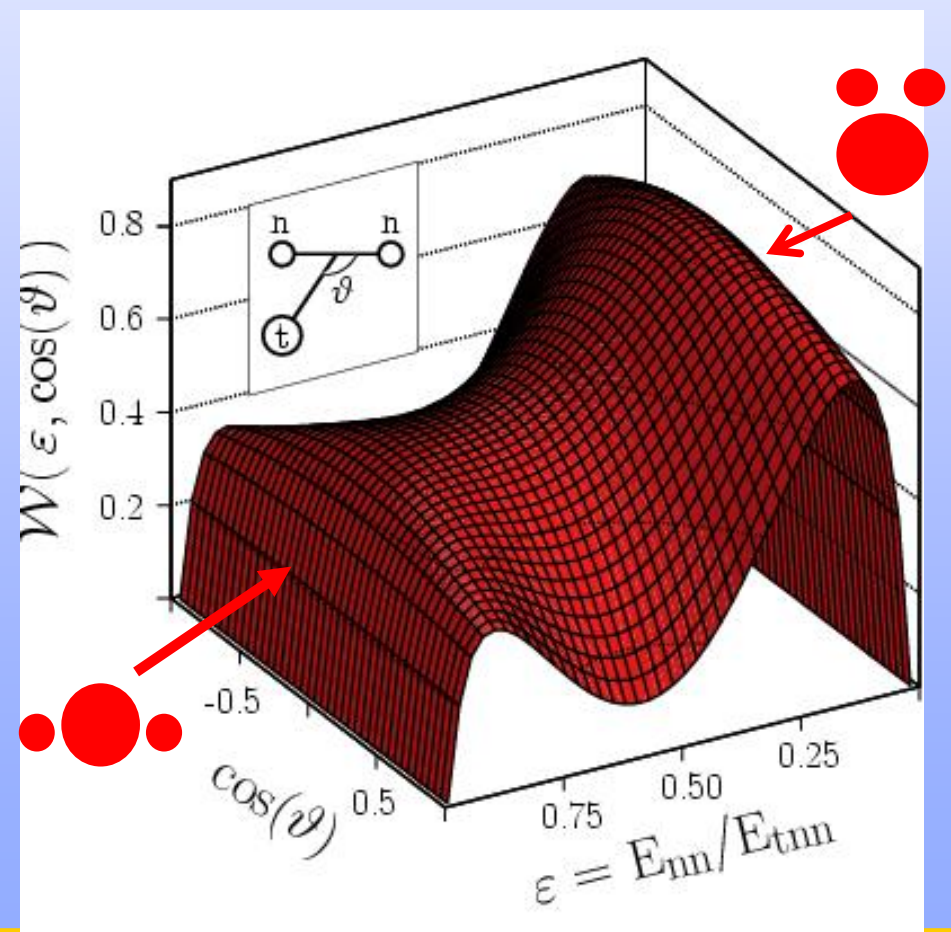




\section{Neutron skins in $\mathrm{Na}$-isotopes}

\section{Total interaction cross sections measured at $950 \mathrm{MeV} / \mathrm{u}{ }^{\mathrm{A} N a} \rightarrow \mathrm{C}$}

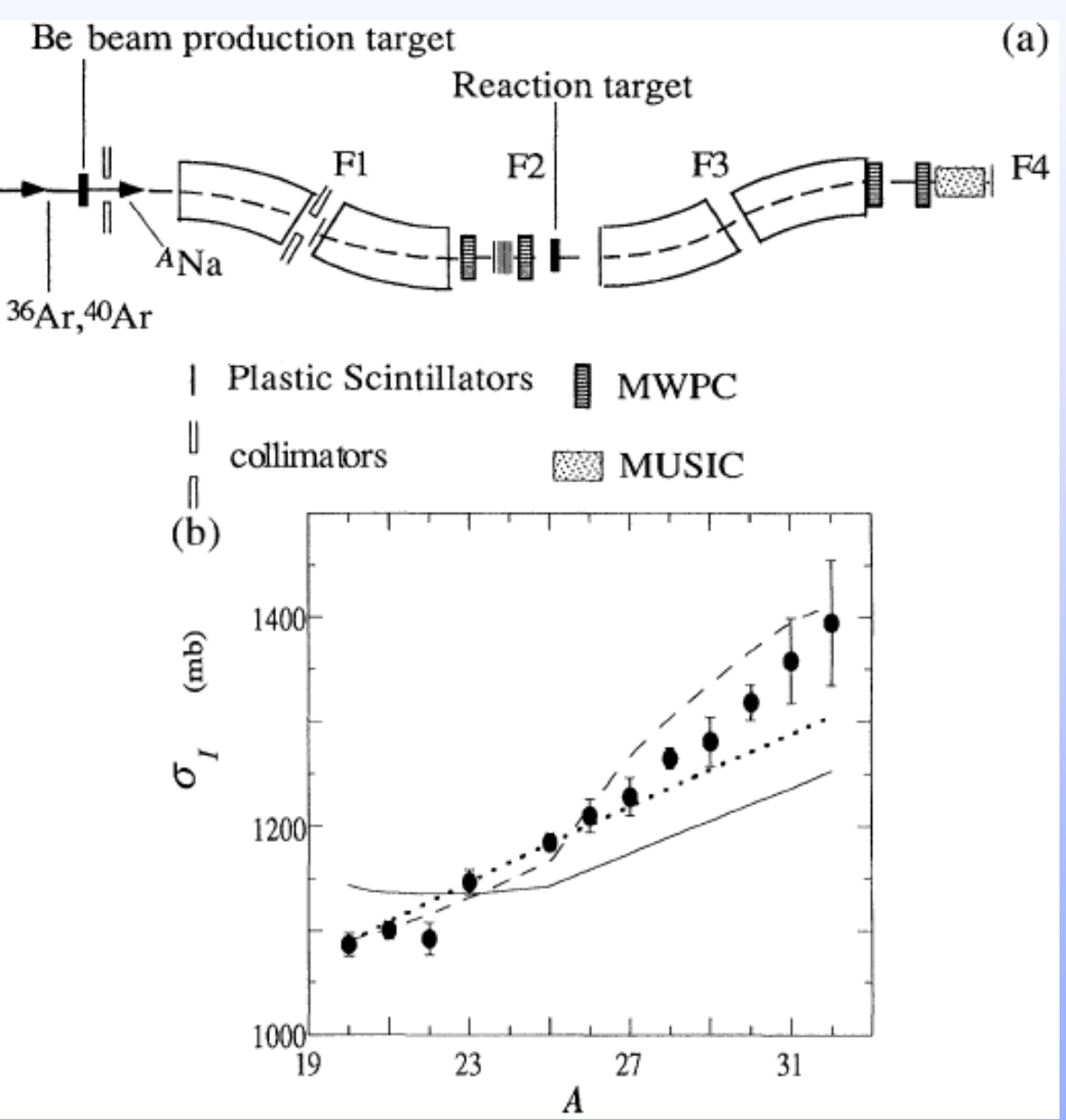

T. Suzuki et al., Phys. Rev. Lett. 75 (1995) 3241

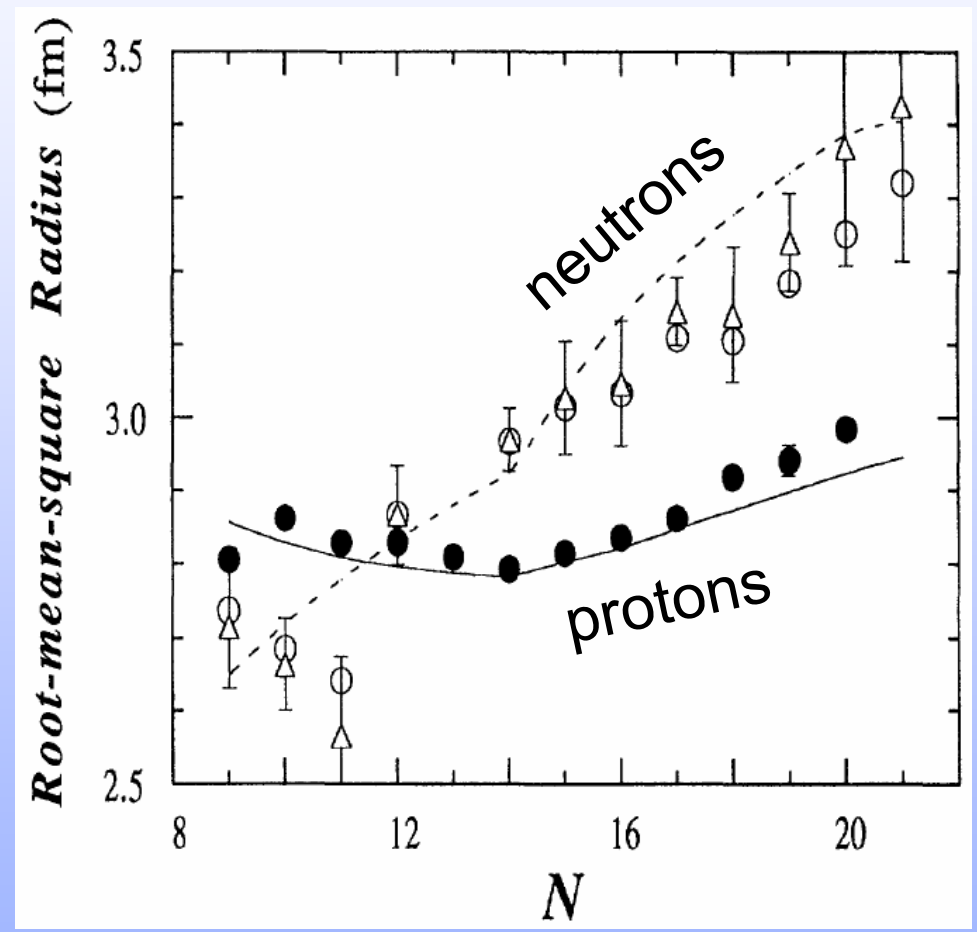

RMS charge radii from isotope-shift measurements, e.g. G. Huber et al., Phys. Rev. C 18 (1978) 2342 


\section{Stable, skin and halo nuclei}

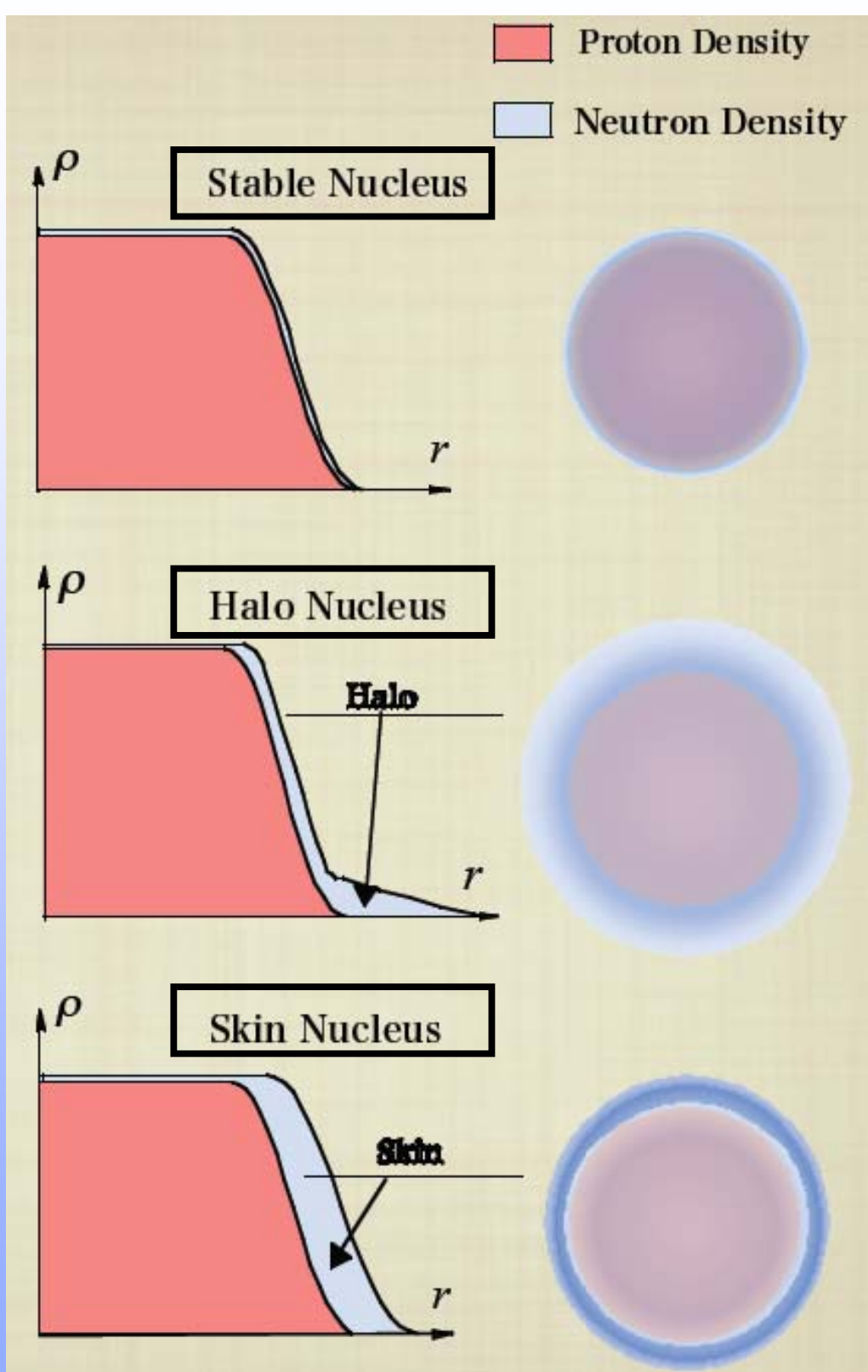




\section{Terra incognita: lithium isotopes beyond the drip-line}

$\sim 300 \mathrm{MeV} / \mathrm{u}{ }^{11} \mathrm{Li},{ }^{14} \mathrm{Be}+$ liq. $\mathrm{H}_{2} \rightarrow{ }^{9} \mathrm{Li}+\mathrm{n},{ }^{11} \mathrm{Li}+\mathrm{n},{ }^{11} \mathrm{Li}+2 \mathrm{n}$

\section{Newly observed ${ }^{12} \mathrm{Li}$ and ${ }^{13} \mathrm{Li}$}

Previous results confirmed: ${ }^{10} \mathbf{L i}$ is known as virtual s-state $(a=-22 \mathrm{fm})$ with an

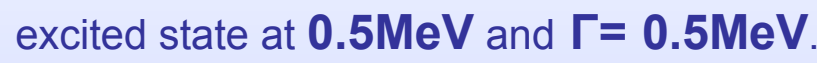

${ }^{12} \mathbf{L i}$ is observed as a virtual s-state with scattering length $a=-11 \mathrm{fm}$
${ }^{13} \mathbf{L i}$ is seen as a broad 3-body resonance state at $\mathbf{1 . 5} \mathbf{M e V}$.
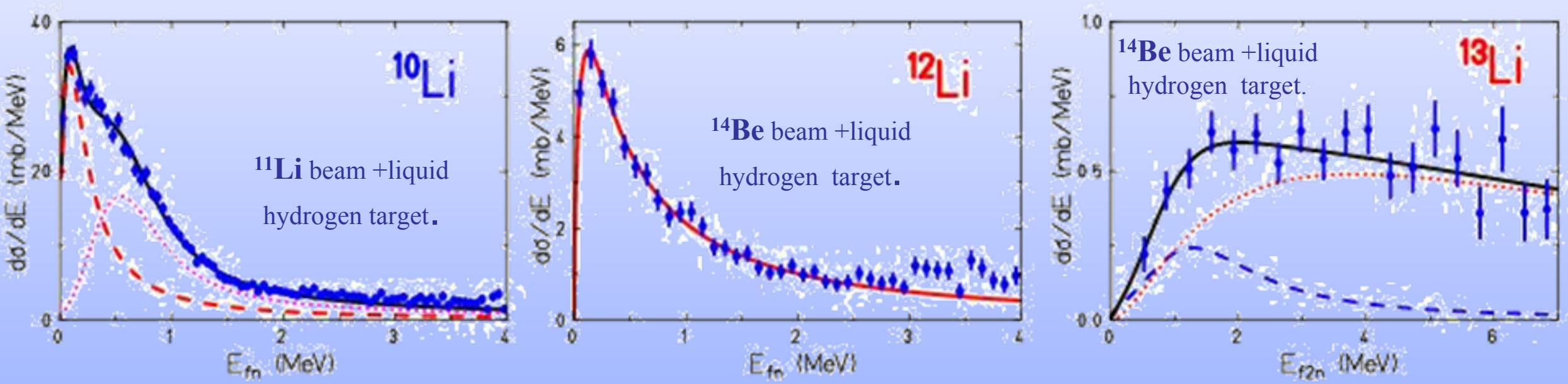

Resonance state

Correlated background

(from nn-correlations in initial bound-state wave function)

Yu. Aksyutina et al., publication in preparation 


\section{Some links to nuclear astrophysics}




\section{Challenge: understand solar system element and mass abundances}

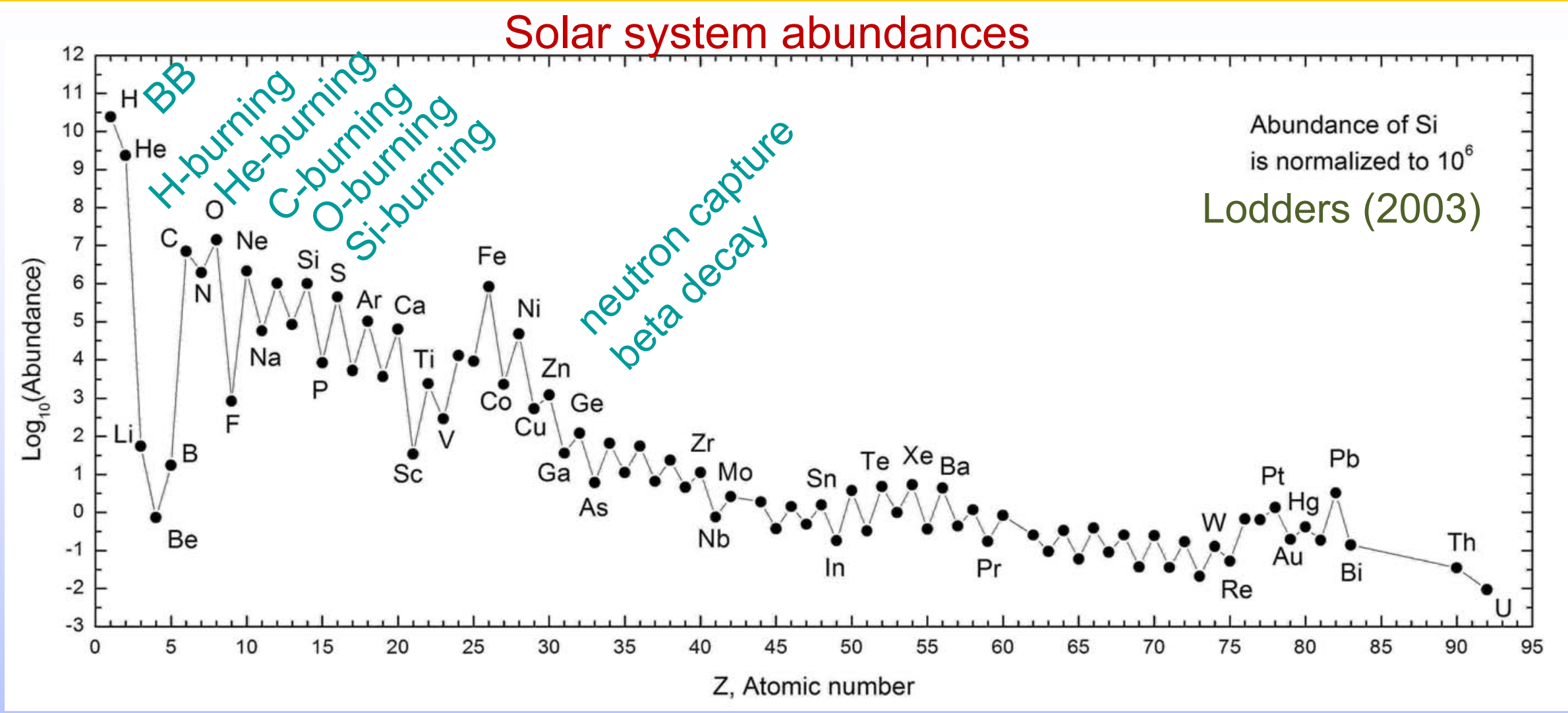

Where, when and how are the elements produced?

Understand the observed distribution, qualitatively and quantitatively!

Why no elements $Z>92$, why no masses $A>240$ ? 


\section{Formation of heavy elements by s- and r-process}

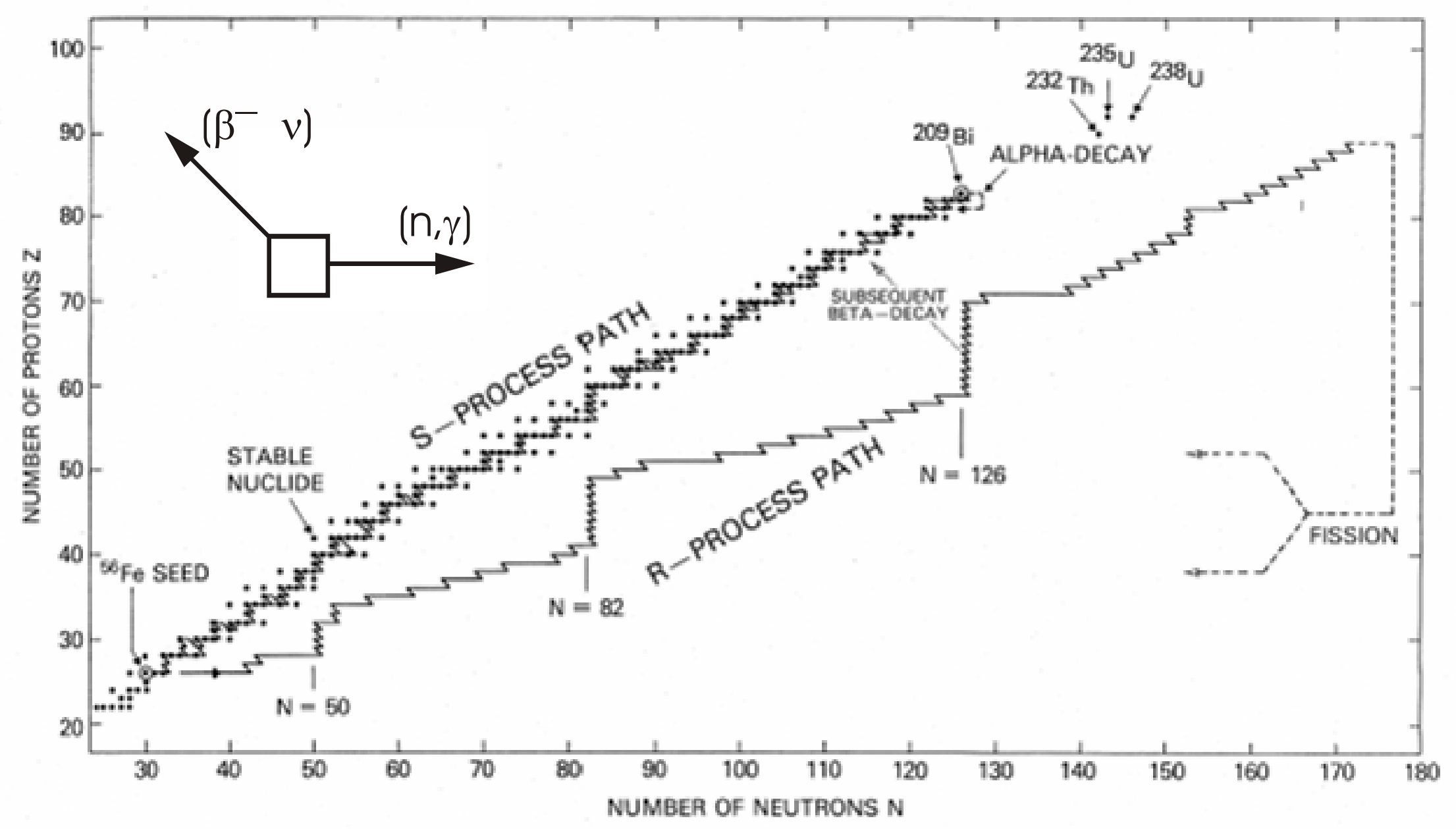

- s-process terminates at ${ }^{209} \mathrm{Bi}$

- $r$-process produces the heaviest elements $(T h, U)$

- $p$-process produces $\sim 30 \mathrm{n}$-deficient isotopes, which cannot be formed by s- or r-process 


\section{Explanation of s-process abundance maxima}

$\mathbf{N}_{\mathbf{A}} \propto \frac{1}{\langle\boldsymbol{\sigma}\rangle_{\mathbf{A}}} \Leftrightarrow \begin{aligned} & \text { small } n \text {-ca } \\ & \text { vice versa }\end{aligned}$
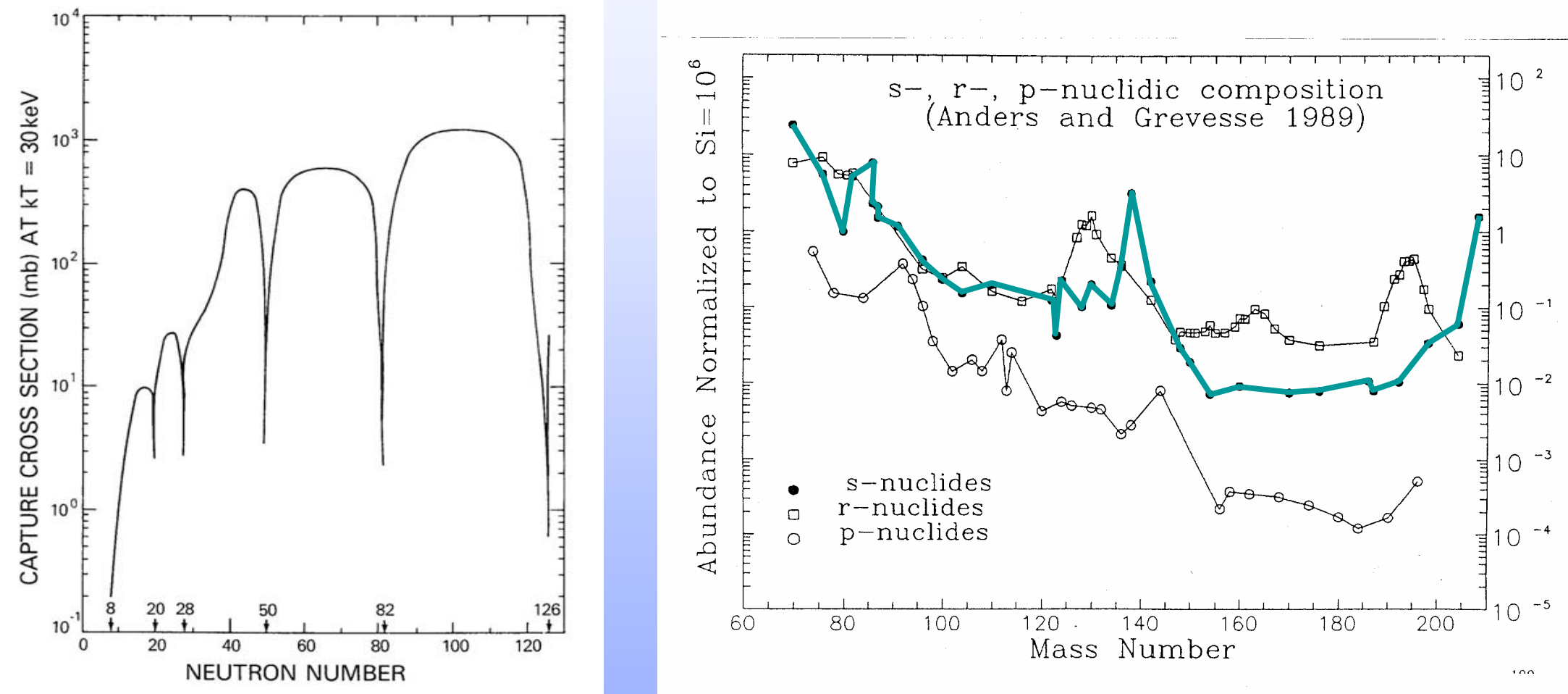

- Temperature-averaged n-capture cross sections needed!

- Near stability 


\section{beta-decay to bound final states}

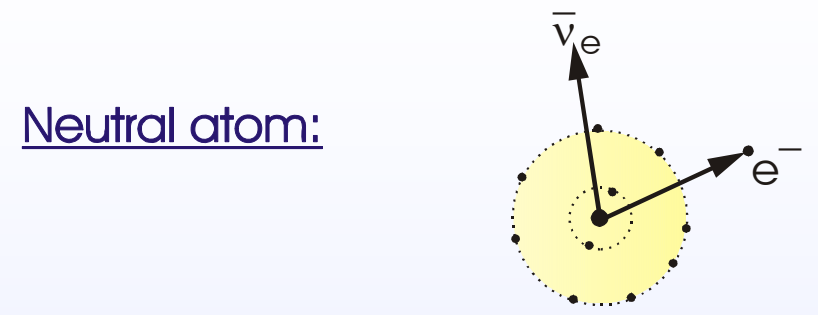

Continuum-state $\beta$-decay

Bare nucleus:

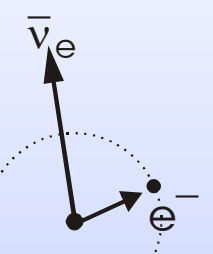

Bound-state $\beta$-decay

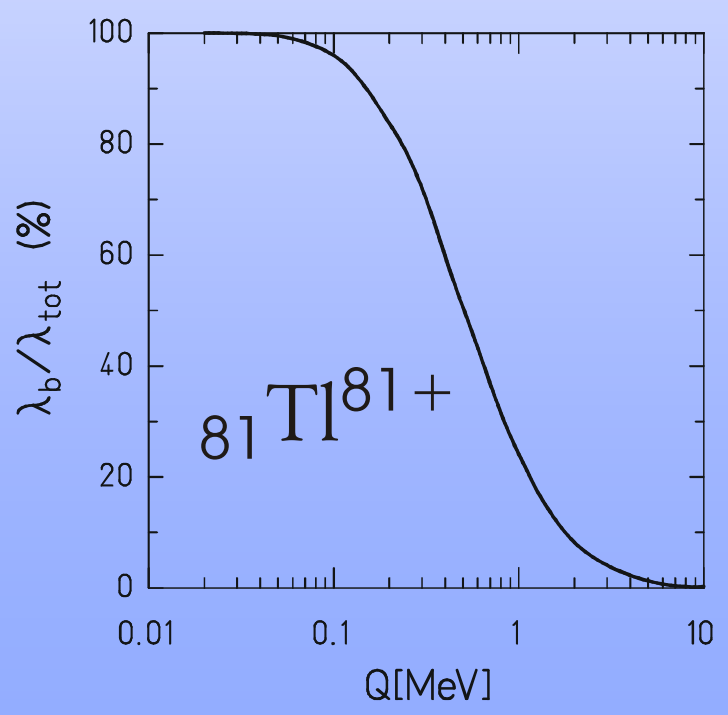




\section{Bound-state beta decay of ${ }^{207} \mathrm{~T}^{81+}$}

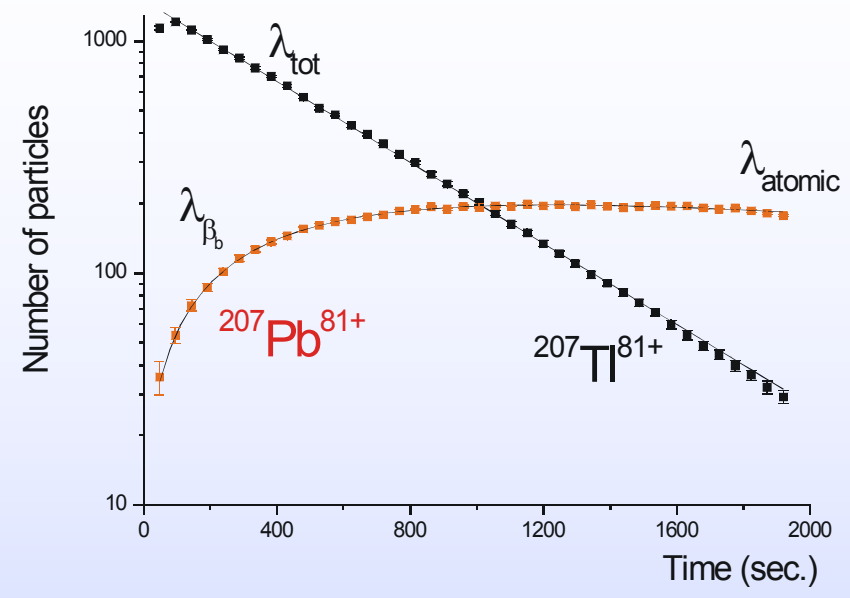

$$
\begin{aligned}
& \text { Half-life } \quad T_{1 / 2}=271 \pm 2 \mathrm{sec} \\
& \text { Branching } \beta_{\mathrm{b}} / \beta_{\mathrm{C}}=0.224 \pm 0.004 \\
& \text { Q-value } \quad Q_{\beta \mathrm{b}}=1507 \pm 8 \mathrm{keV}
\end{aligned}
$$

Calculation by

K. Takahashi and K. Yokoi, ADNDT 36 (1987)

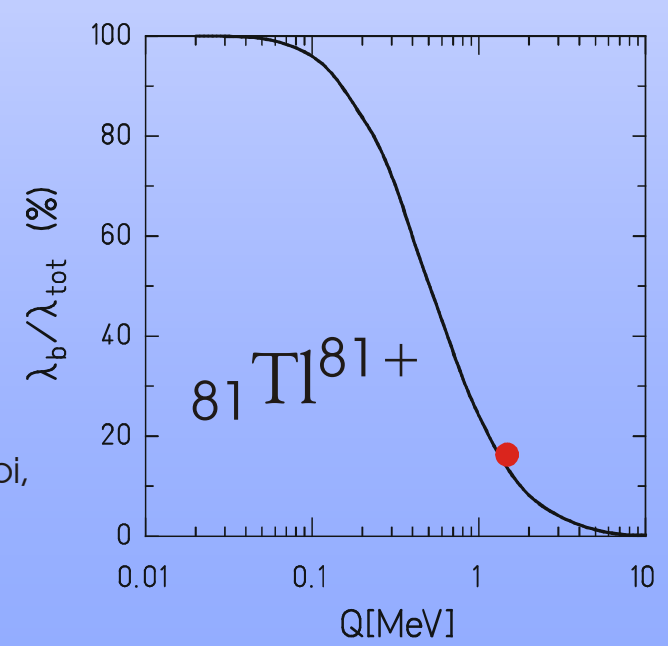




\section{r-process}

Assumption: $(\mathrm{n}, \gamma) \leftrightarrow(\gamma, \mathrm{n})$ rate equilibrium

$\lambda_{\gamma \mathbf{n}} \propto \frac{T^{3 / 2}}{N_{n}} \exp \left(-\frac{Q_{n}}{k T}\right) \cdot \lambda_{n \gamma}$

Example: $\mathrm{N}_{\mathrm{n}}=10^{24} / \mathrm{cm}^{3}, \mathrm{~T}_{9}=1$

$$
\rightarrow Q_{n}=2 \mathrm{MeV}
$$

Neutron capture processes stall, and nucleus „waits“ for $\beta^{-}$-decay:

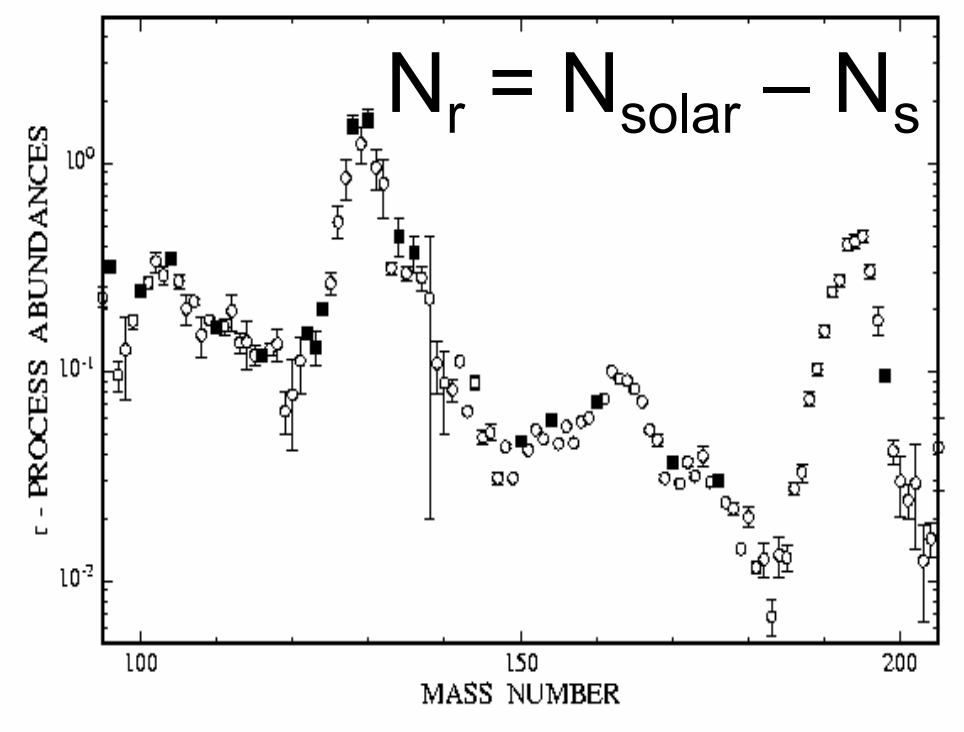

${ }_{z} X \rightarrow{ }_{z+1} X+e^{-}+v_{e}$

$\rightarrow$ for every element, there is a so-called „waiting point“

$\rightarrow$ r-process path determined by mass differences

$\rightarrow$ abundances determined by half-lives 


\section{Uncertainty between models and nuclear properties}

Astrophysics modified

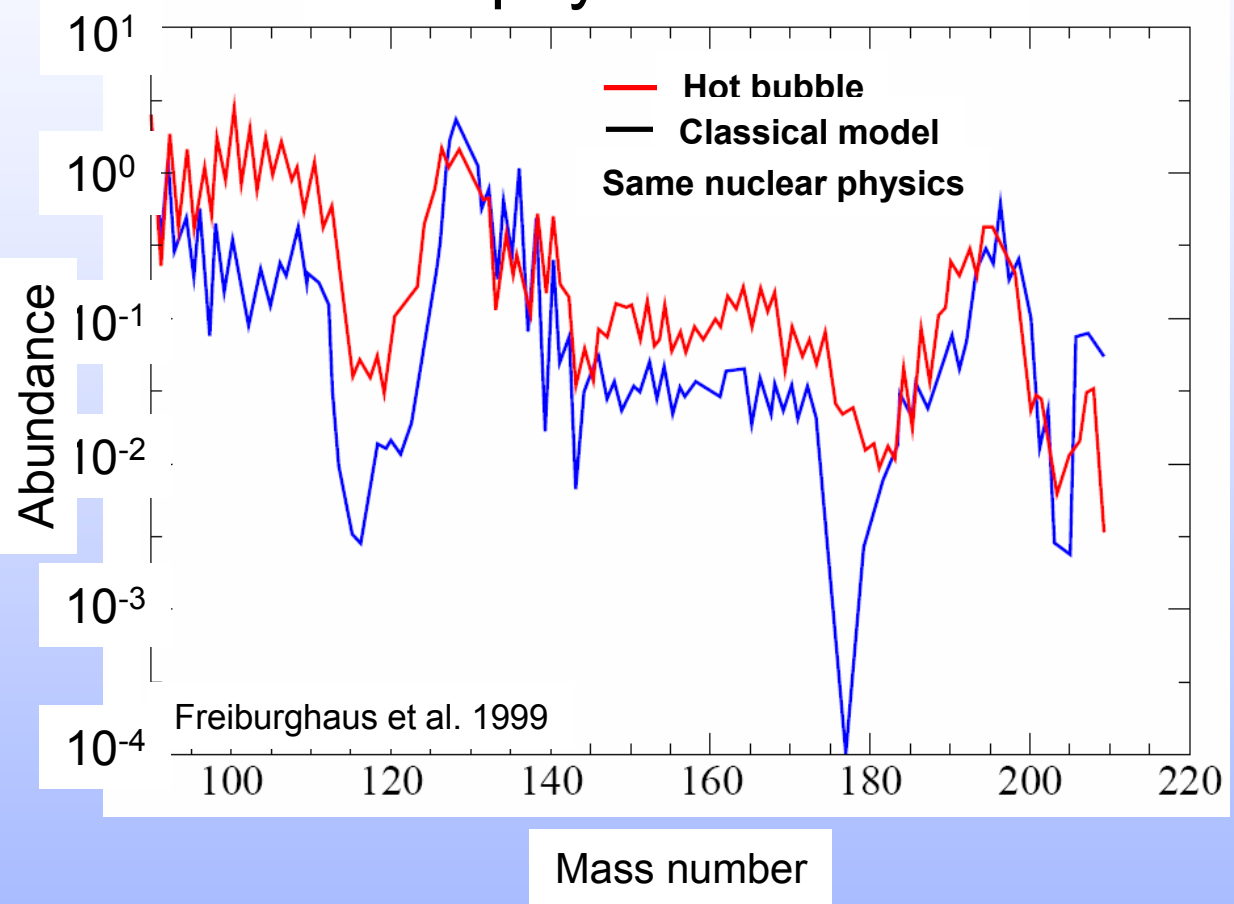

Nuclear physics modified

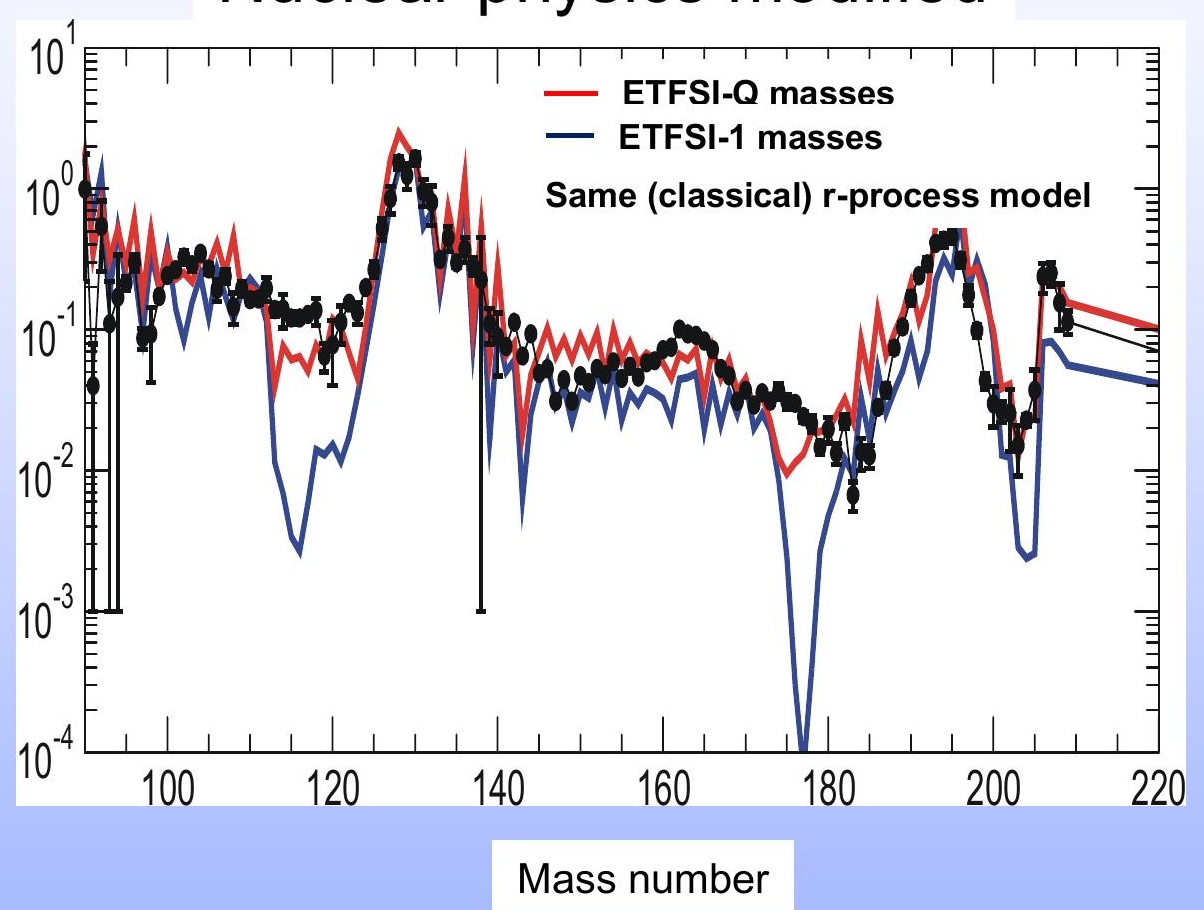

Are the fine details a reflection of the stellar site or of nuclear physics input? 


\section{Importance of mass measurements}

- Nuclei far-off stability may show different phenomena than nuclei close to stability (magic numbers, shell quenching)

- Extrapolation of mass models to regions far from stability may introduce errors

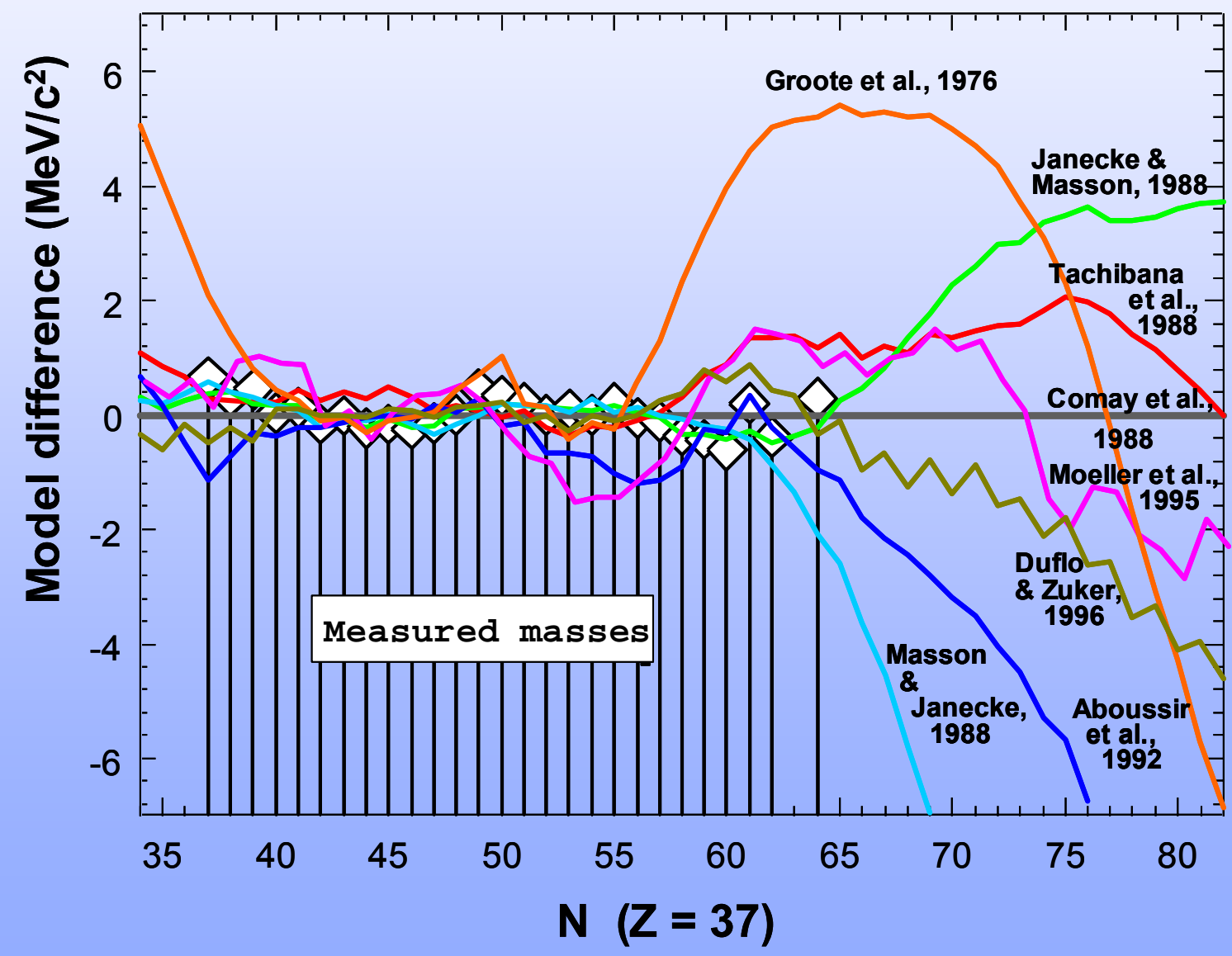

D. Lunney, 2001 


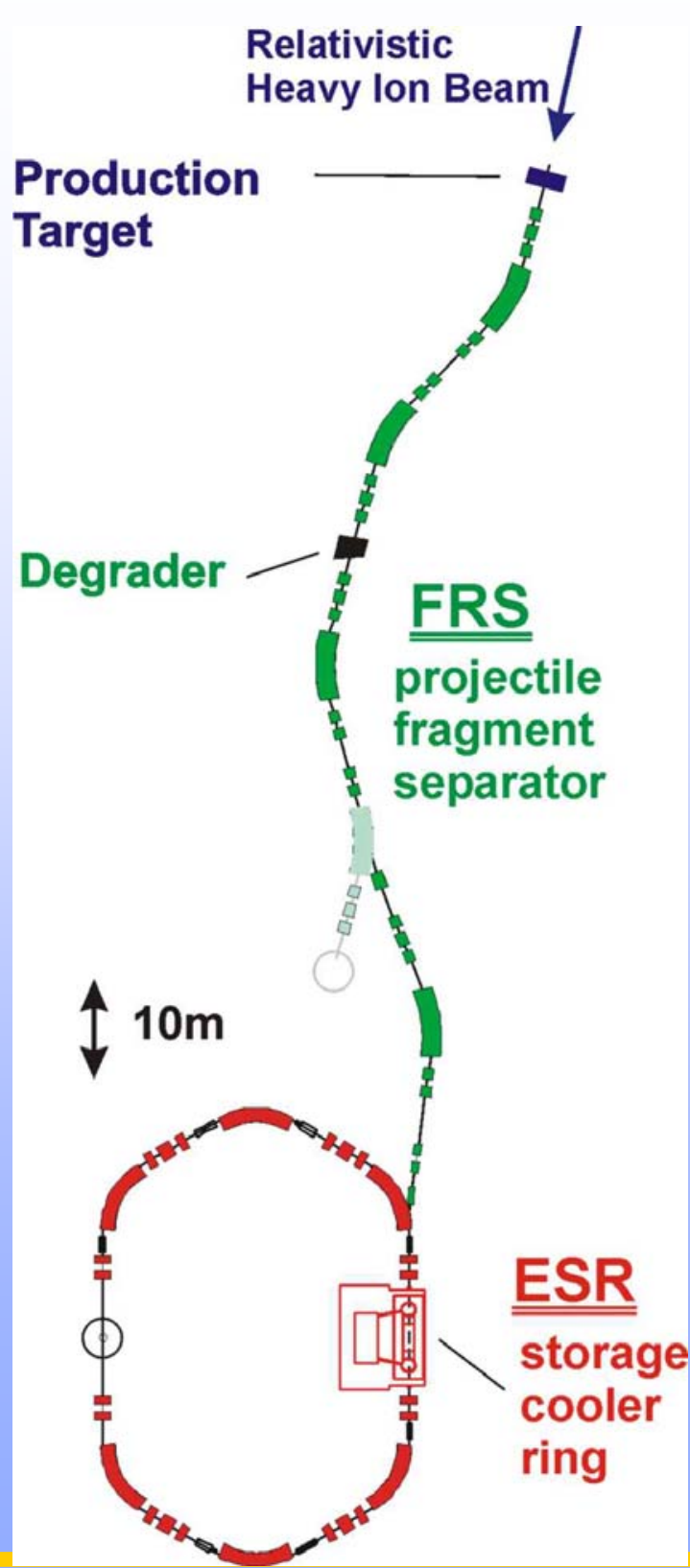

Production:

* Primary beams:

$\mathrm{H}$.....U, 100...1000MeV/u

* Reaction mechanisms:

Projectile fragmentation, ED and fission

* Yields:

$\sim 105 /$ s.... 10 $-5 / \mathrm{s}$ (=1/day)

* Ionic charge states: bare, $\mathrm{H}$-, He-, Li-like

Separation:

B $\rho$-Analysis

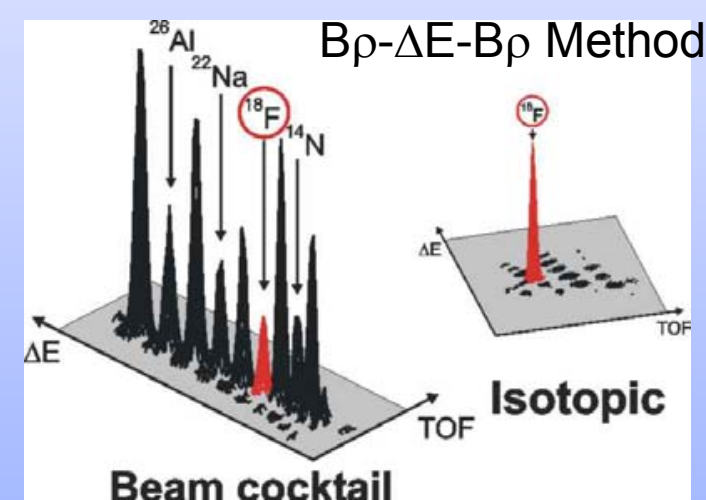

\begin{tabular}{|l|c|c|}
\hline & IMS & $\begin{array}{c}\mathrm{SM} \\
\mathrm{S}\end{array}$ \\
\hline $\begin{array}{l}\text { Mass resolving } \\
\text { power } \mathrm{m} / \Delta \mathrm{m}_{\mathrm{FWHM}}\end{array}$ & $1 \cdot 10^{5}$ & $1-2 \cdot 10^{6}$ \\
\hline $\begin{array}{l}\text { Mass } \\
\text { accuracy }\end{array}$ & $\sim 100 \mathrm{keV}$ & $\sim 30 \mathrm{keV}$ \\
\hline $\begin{array}{l}\text { Accessible } \\
\text { half-lives }\end{array}$ & $>10 \mu \mathrm{s}$ & $>1 \mathrm{~s}$ \\
\hline $\begin{array}{l}\text { Sensitivity } \\
\text { single } \\
\text { ions }\end{array}$ & $\begin{array}{c}\text { single } \\
\text { ions }\end{array}$ \\
\hline
\end{tabular}

Storage:

${ }^{*}$ Fast injection (bunch length 400ns)

* Storage times: minutes .... hours

* Cooling: - stochastic (pre-)cooling - electron cooling 


\section{Mass Measurements at the Ring Branch}

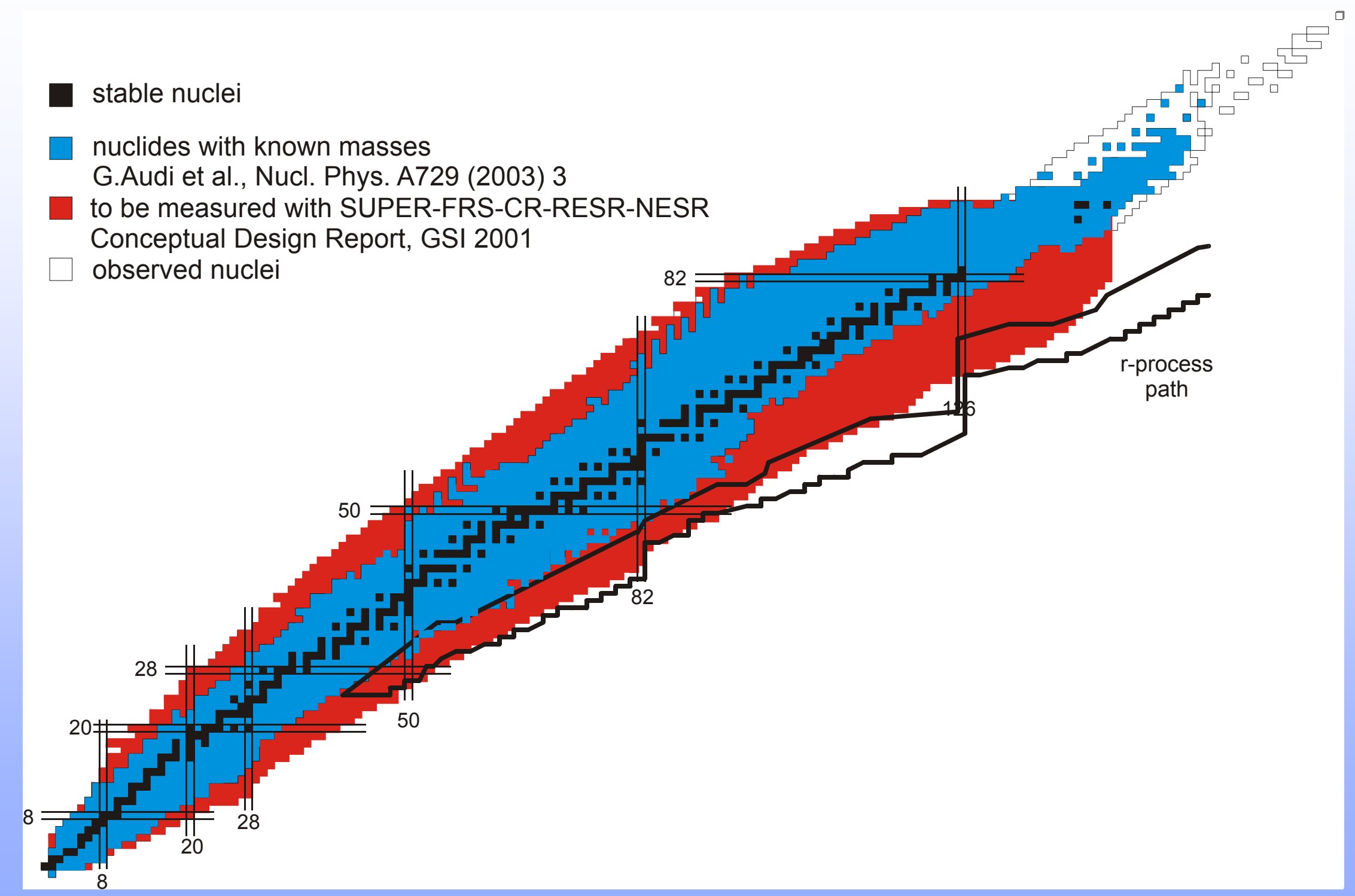

Yu. Litvinov

Christoph Scheidenberger, GSI and University Gießen

Exotic nuclei 


\section{Future opportunities at FAIR}




\section{FAIR - International Facility for Antiproton and Ion Research}

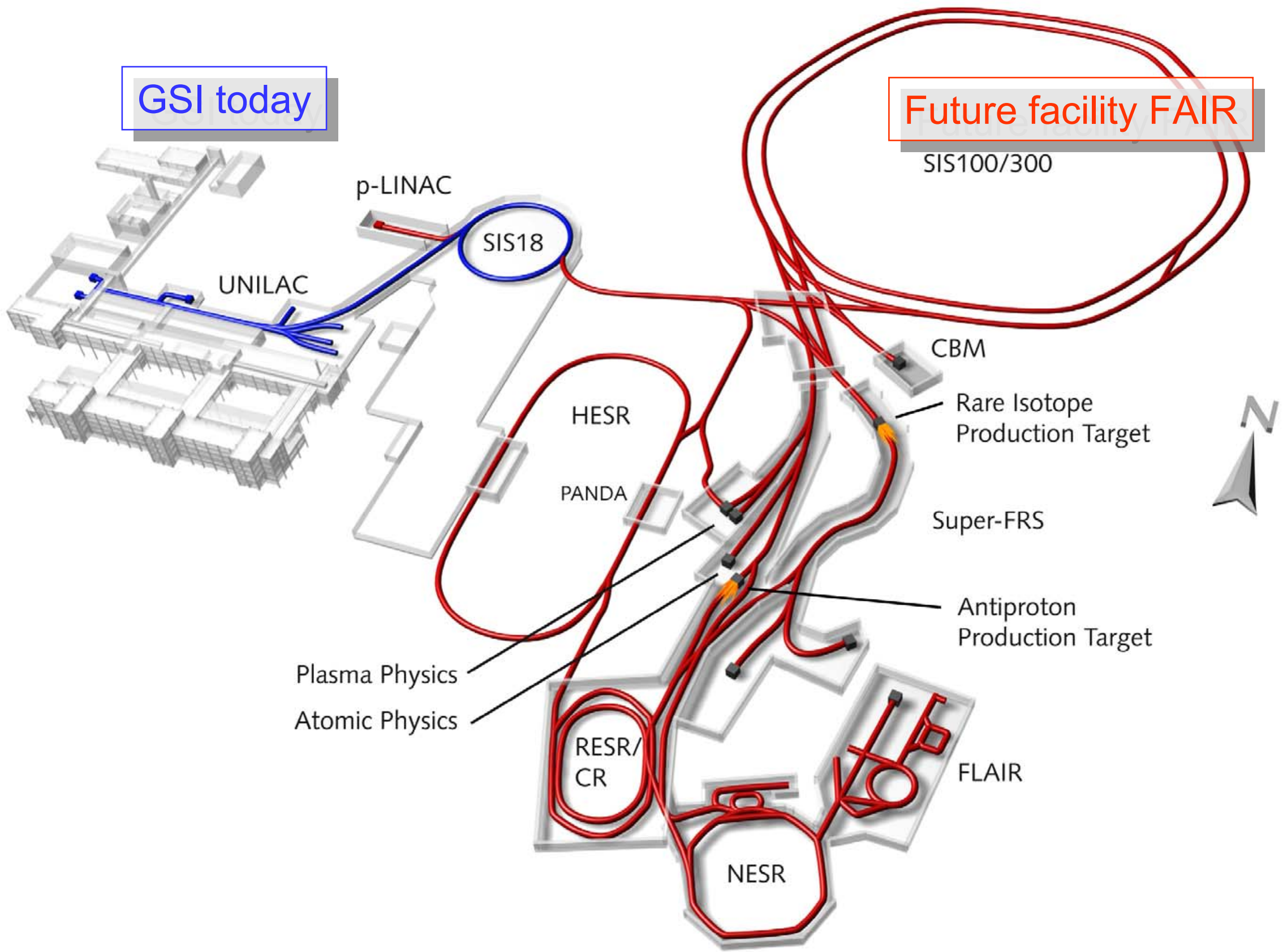




\section{Challenges and future opportunities}
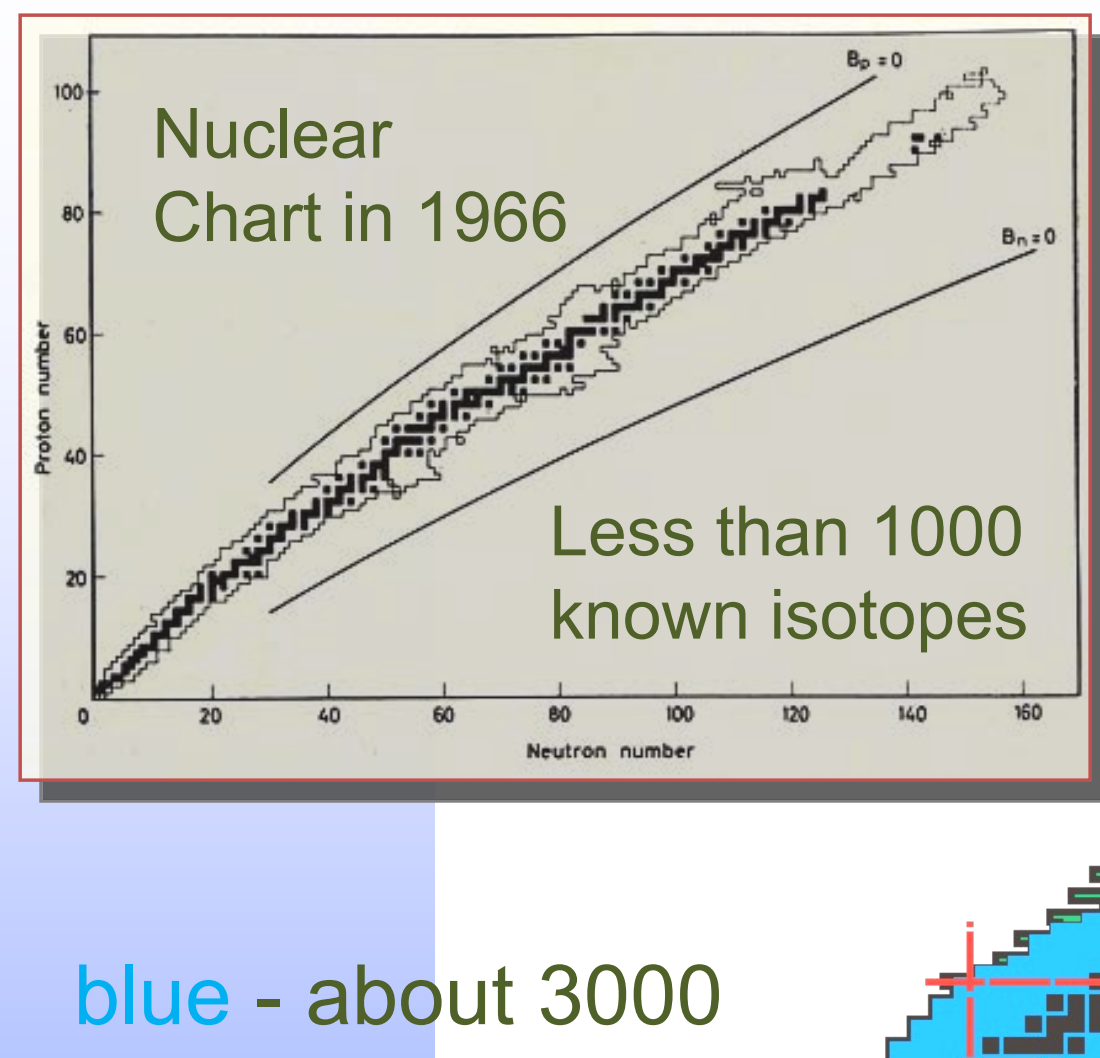

known isotopes

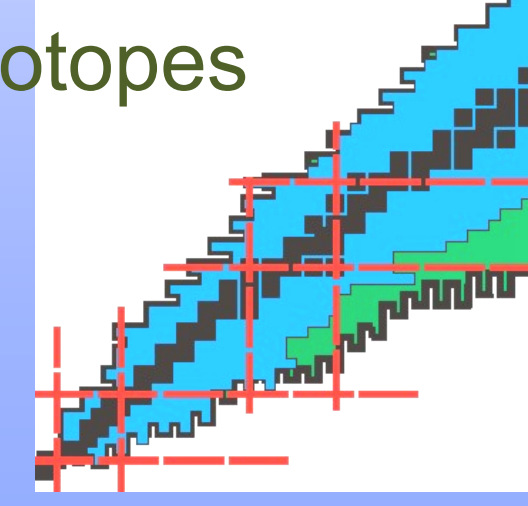

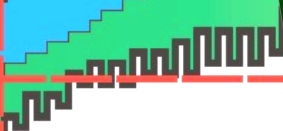

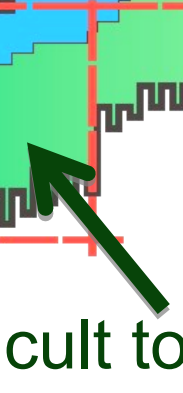
More difficult to
produce and separate
( 1 in $10^{18}$ atoms) More difficult to
produce and sep
( 1 in $10^{18}$ atoms)

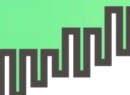

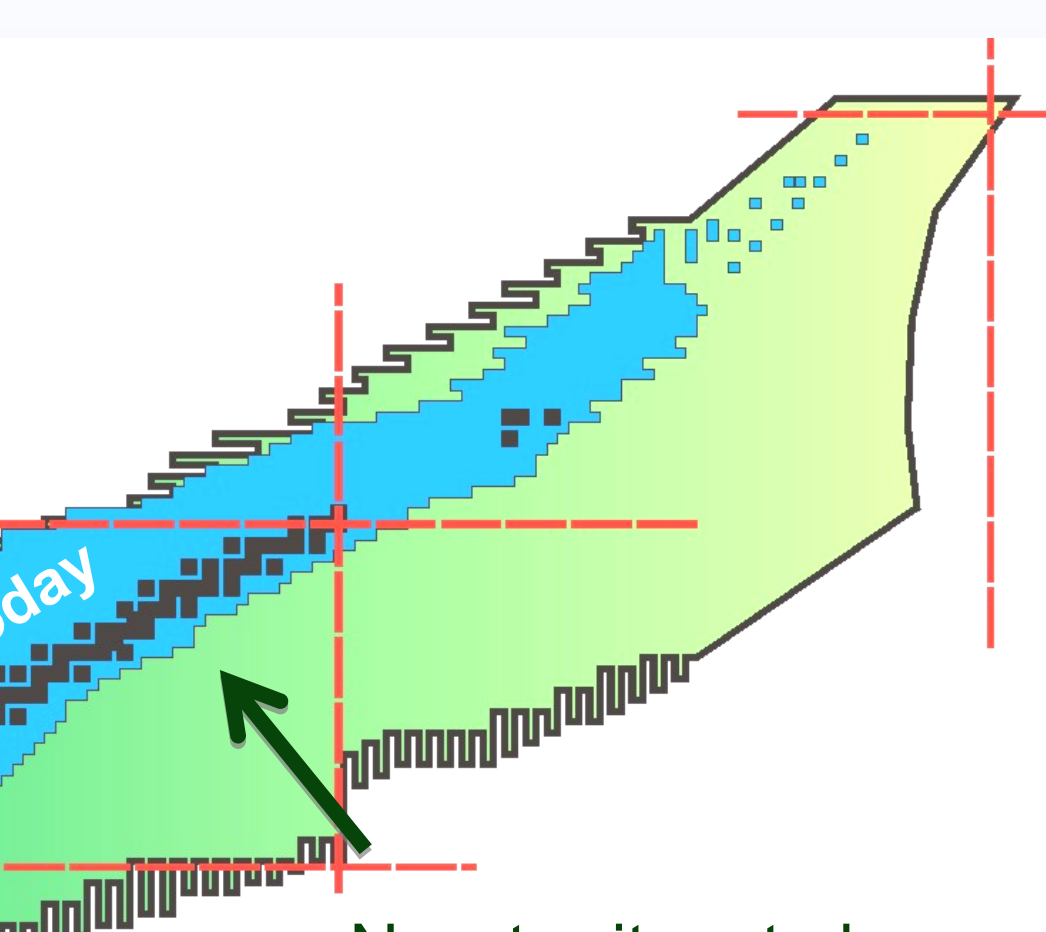

New territory to be explored with nextgeneration rare isotope facilities (BigRIPS, SuperFRS, FRIB Separator) 


\section{Rate estimates}

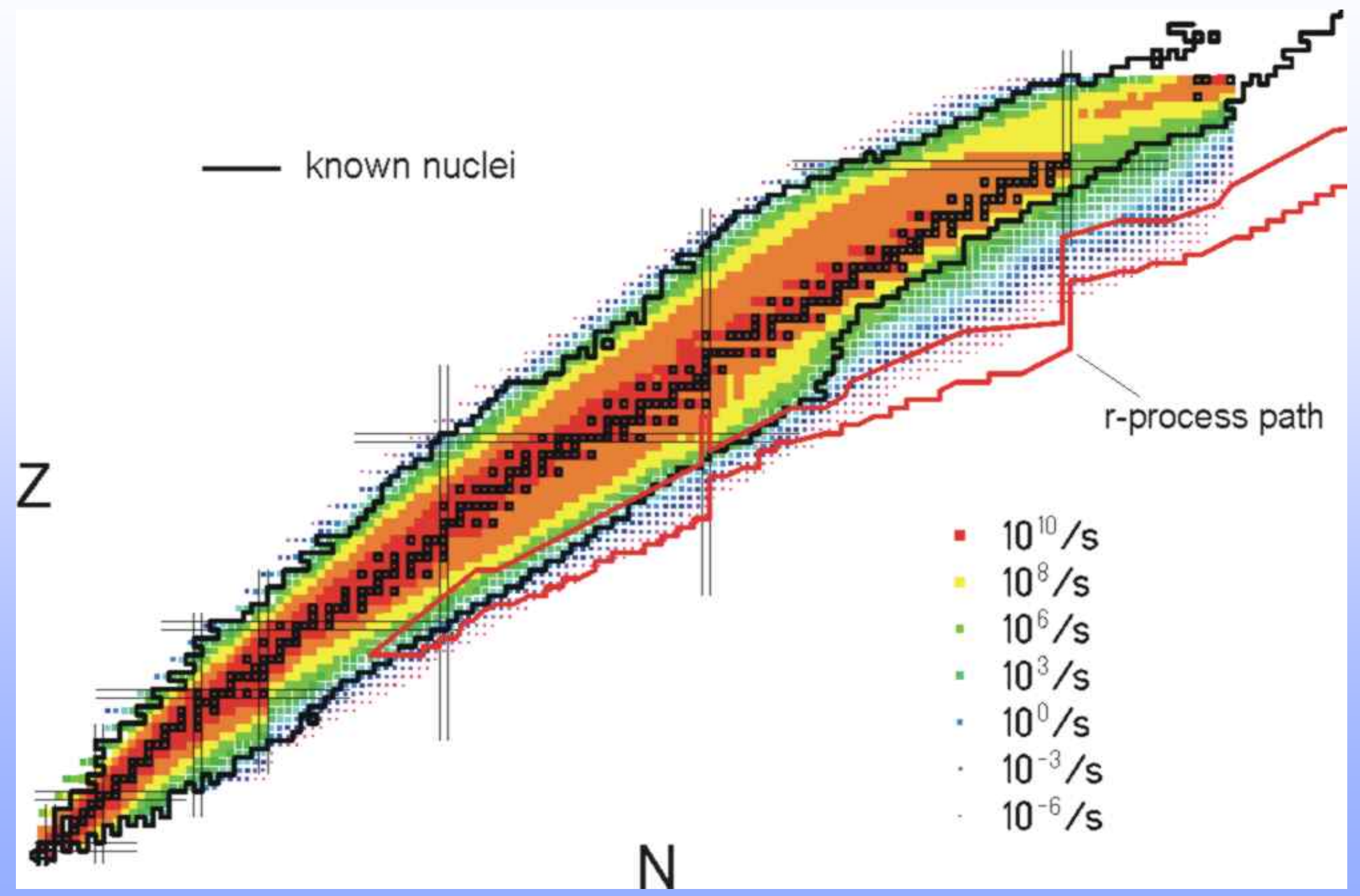




\section{Thank you for attention!}

\section{Enjoy the school and the NIC conference!}




\section{End}

